\title{
Molecular Droplets vs. Bubbles: effect of curvature on surface tension and Tolman length
}

\author{
Jun Wen ${ }^{1,2, * *}$, Daniele Dini ${ }^{2}$, Haibao Hu${ }^{1}$, Edward R. Smith ${ }^{3}$ \\ ${ }^{1}$ School of Marine Science and Technology, Northwestern Polytechnical University, Xi'an \\ 710072, China. \\ ${ }^{2}$ Department of Mechanical Engineering, Imperial College London, Exhibition Road, South \\ Kensington, London SW7 2AZ, United Kingdom \\ ${ }^{3}$ Department of Mechanical and Aerospace Engineering, Brunel University London, \\ Uxbridge, Middlesex UB8 3PH, United Kingdom
}

\begin{abstract}
Droplets and bubbles are thought to be two sides of the same coin; this work determines how true this is at the molecular scale. Stable cylindrical nanodroplets and nanobubbles are obtained in Molecular Dynamics (MD) simulations with three-phase contact lines pinned by alternate hydrophobic and hydrophilic patterns. The surface tension and Tolman length for both types of curved interfaces are obtained with the Kirkwood-Buff method, based on the difference between normal and tangential pressure components. Both bubble and droplet cases are compared to the flat interface case for reference. Results show that the surface tension decreases linearly while the Tolman length increases linearly with the gas/liquid density ratio. By running a careful parameter study of the flat interface over a range of densities, the effect of density ratio can be corrected isolating the effects of curvature on the surface tension and Tolman length. It is found that such effects start to be seen when the equimolar curvature radius goes down to 20 reduced LJ units. They have the same magnitude but act with opposite signs for nanodroplet and nanobubble interfaces. Considering effects of density ratio and curvature, a fitted Tolman equation was obtained which predicts the surface tension of a curved interface. Results obtained by the fitted Tolman equation agree well with those obtained by the MD simulations except at very small curvature radius ( $<10$ reduced LJ units) due to the accumulation of the curvature dependence of the Tolman length.
\end{abstract}

\footnotetext{
** Corresponding author: j.wen17@imperial.ac.uk; currently at wejun@mail.nwpu.edu.cn
} 


\section{INTRODUCTION}

The surface tension is an important interfacial property of an inhomogeneous system and is of interest in the study of many areas of fluid dynamics, including droplet/bubble nucleation, ${ }^{1,2}$ surface wetting, ${ }^{3-}$ ${ }^{5}$ and the moving contact line $e^{6}$. It results from the intermolecular forces at the interface between two bulk phases. Thus, the surface tension is directly related to pressure tensors, which in dense fluids is predominantly due to the intermolecular forces. The pioneering work on the surface tension dates back to the nineteenth century when the Young-Laplace equation ${ }^{7-12}$ was derived at the macroscale. For a spherical interface, it can be stated as:

$$
\Delta P=\frac{2 \gamma}{R}
$$

where $\Delta P$ is the pressure drop across a spherical interface, $\gamma$ is the surface tension and $R$ is the curvature radius of the interface. This equation shows great accuracy at the macroscale and has been used for decades. In an inhomogeneous system, two bulk phases (such as liquid and vapour) transit to each other through a layer where the physical properties change dramatically. But there is not an exact physical interface which separates the two bulk phases. Mathematically, two interfaces which separate the two phases explicitly were defined by Gibbs. One is the equimolar surface where the superficial number density of particles (molecules) vanishes. The other one is the surface of tension where the surface tension is supposed to act. We can see that different molecules would contribute equally to the equimolar surface if they are at the same position, as the contribution to the equimolar surface is exclusively determined by the number of interacting molecules. On the other hand, the surface of tension depends on the intermolecular forces and therefore on the type of molecules. Thus, contributions of different types of molecules to the surface of tension differ from each other, so the equimolar surface and the surface of tension normally do not coincide. ${ }^{7-11}$ Tolman $^{13,14}$ was among the first of several researchers who investigated the distance between the equimolar surface and the surface of tension which was later named after him as the Tolman length $(\delta)$. At the macroscale, the curvature radius of an interface is much larger than the intermolecular interaction distance. The stress state of a molecule on a curved interface can be approximated as the same as the one on a flat interface. Thus, the curvature does not show appreciable effect on surface tension and it can be regarded as constant. However, when the curvature radius of the interface reduces to the scale of the molecular interaction, the curvature starts to show a strong effect on the surface tension. Based on Gibbs's work, Tolman has derived an equation to show the curvature dependence of the surface tension. ${ }^{13,14}$ By neglecting terms $\mathcal{O}(\delta / R)$ and assuming that $\delta$ is constant, the equation for a spherical droplet interface reads:

$$
\frac{\gamma_{R}}{\gamma_{\infty}}=\frac{1}{1+\frac{2 \delta}{R}}
$$

where $\gamma_{R}$ and $\gamma_{\infty}$ are the surface tension for an spherical droplet interface with a radius of $R$ and a flat interface, respectively. However, recent studies have shown that $\delta$ also has a curvature dependence ${ }^{8,9}$, 
15-19. Neglecting the terms $\mathcal{O}(\delta / R)$ may also have an appreciable effect on the results $18,20,21$. Mathematically, the Tolman length is normally defined as $\delta=R_{\mathrm{e}}-R_{S}$, where $R_{\mathrm{e}}$ and $R_{\mathrm{S}}$ are the curvature radii of the equimolar interface and the surface of tension, respectively. ${ }^{8,9}$ Such definitions of the Tolman length, including Eq. (2), were derived based on a system with a liquid droplet surrounded by a vapour phase ${ }^{13}$ with the curvature radius for a droplet defined as being positive. This equation can be extended to systems with bubbles submerged in liquid by defining negative curvature radii for bubbles. ${ }^{11,22}$ Based on Eq. (2), a constant Tolman length leads to opposite effects on the surface tension for droplets and bubbles. ${ }^{23}$ Although the curvature dependence of surface tension and the Tolman length for nanodroplets has drawn much attention in numerical simulations, ${ }^{19,24-26}$ studies on nanobubbles are rare due to computational expense and difficulties in forming stable nanobubbles.

In this study, a statistic mechanical approach, based on MD simulations, was adopted to simulate the surface tension and Tolman length of flat, nanodroplet and nanobubble interfaces. The research systems and methods adopted are introduced in section II. In section III, basic results for flat, nanodroplet and nanobubble interfaces are presented first. The effect of the gas/liquid density ratio $\left(\rho_{\mathrm{G}} / \rho_{\mathrm{L}}\right.$, where $\rho_{\mathrm{G}}$ and $\rho_{\mathrm{L}}$ are the gas density and liquid density, respectively) on the surface tension and Tolman length for a flat interface is discussed next. Then, the results of surface tension and Tolman length for nanodroplet and nanobubble interfaces are compared and discussed. The curvature dependence of the surface tension and Tolman length is obtained. In the end of the third section, fitted Tolman equations considering the effect of gas/liquid density ratio and curvature are obtained. We conclude in section IV by highlighting the key findings of this work and their implication for practical applications. 


\section{METHODOLOGY}

\section{A. Theoretical investigation of the way for calculating the surface tension}

The surface tension of the flat, cylindrical nanobubble and nanodroplet interfaces were simulated with classical MD simulations. The equations for calculating the surface tension with pressure tensors of a flat interface are: ${ }^{25}$

$$
\begin{gathered}
\gamma_{\mathrm{F}}=\int_{y_{\mathrm{G}}}^{y_{\mathrm{L}}}\left(P_{\mathrm{N}}-P_{\mathrm{T}}\right) \mathrm{d} y \\
\gamma_{\mathrm{F}} y_{\mathrm{s}}=\int_{y_{\mathrm{G}}}^{y_{\mathrm{L}}} y\left(P_{\mathrm{N}}-P_{\mathrm{T}}\right) \mathrm{d} y
\end{gathered}
$$

where $\gamma_{\mathrm{F}}$ is the surface tension, $P_{\mathrm{N}}$ and $P_{\mathrm{T}}$ are the normal and tangential pressure components, respectively. $y$ is the direction normal to the gas/liquid interface, $y_{\mathrm{G}}$ and $y_{\mathrm{L}}$ are two locations deep inside the bulk gas and the bulk liquid regions, respectively and $y_{S}$ is the location of the surface of tension where the surface tension acts.

The location of the equimolar surface can be obtained by the following equation:

$$
\int_{y_{\mathrm{G}}}^{y_{\mathrm{e}}} \rho_{\mathrm{G}} \mathrm{d} y+\int_{y_{\mathrm{e}}}^{y_{\mathrm{L}}} \rho_{\mathrm{L}} \mathrm{d} y=\int_{y_{\mathrm{G}}}^{y_{\mathrm{L}}} \rho_{\mathrm{y}} \mathrm{d} y
$$

where $\rho_{\mathrm{G}}$ and $\rho_{\mathrm{L}}$ are the molecule number density in the bulk gas and bulk liquid regions, respectively. $\rho_{\mathrm{y}}$ is the molecule number density at location $y . y_{\mathrm{e}}$ is the location of the equimolar surface.

Thus the magnitude of the Tolman length, which is defined as the distance from the surface of tension to the equimolar surface in the direction from liquid to gas, for a flat interface $\left(\delta_{\mathrm{F}}\right)$ can be obtained:

$$
\delta_{\mathrm{F}}=y_{\mathrm{e}}-y_{\mathrm{s}}
$$

In this work, for simplicity we consider two-dimensional examples of both droplets and bubbles, with a small depth in the $\mathrm{z}$ direction between periodic boundary conditions. As a result, the system is a cylindrical interface, so the equations for the surface tension and the curvature radius of the surface of tension based on pressure tensors are deduced following the method similar to that for a spherical interface (see the appendix A for detailed derivation):

$$
\begin{gathered}
\gamma=\int_{R_{\alpha}}^{R_{\beta}} \frac{r}{R_{S}}\left(P_{\mathrm{N}}-P_{\mathrm{T}}\right) d r \\
R_{S}{ }^{2}=\frac{\int_{R_{\alpha}}^{R_{\beta}} r\left(P_{\mathrm{N}}-P_{\mathrm{T}}\right) d r}{\int_{R_{\alpha}}^{R_{\beta}} \frac{1}{r}\left(P_{\mathrm{N}}-P_{\mathrm{T}}\right) d r}
\end{gathered}
$$


where $R_{\alpha}$ and $R_{\beta}$ are deep inside the droplet (or bubble) and outer bulk-gas (liquid) regions, respectively. $\gamma$ and $R_{S}$ are the surface tension and the curvature radius of the surface of tension for both the cylindrical droplets and bubbles. Note here that both curvature radii for bubbles and droplets are defined as being positive in this study. Equations (7) and (8) are used in this study to calculate the surface tension and the curvature radius of the surface of tension. The equimolar curvature radius of the cylindrical droplets and bubbles $\left(R_{\mathrm{e}}\right)$ can be obtained by the following equation ${ }^{27}$ :

$$
R_{\mathrm{e}}{ }^{2}=\frac{\int_{R_{\alpha}}^{R_{\beta}} r^{2} \rho d r}{\int_{R_{\alpha}}^{R_{\beta}} \rho d r}
$$

As the Tolman length is defined as the distance from the surface of tension to the equimolar surface in the direction from liquid to gas in this study, the Tolman length for nanodroplets $\left(\delta_{\mathrm{D}}\right)$ is

$$
\delta_{\mathrm{D}}=R_{\mathrm{e}}-R_{\mathrm{S}}
$$

While the Tolman length for nanobubbles $\left(\delta_{\mathrm{B}}\right)$ is

$$
\delta_{\mathrm{B}}=R_{\mathrm{S}}-R_{\mathrm{e}}
$$

Based on the Gibbs ${ }^{28}$ thermodynamic theory of capillary, a relationship between the surface tension and the Tolman length was deduced by Tolman ${ }^{13}$ for a spherical droplet interface within a one-component two-phase system which was later extended to multicomponent systems by Koenig: ${ }^{29}$

$$
\frac{1}{\gamma_{\mathrm{SD}}} \frac{d \gamma_{\mathrm{SD}}}{d r}=\frac{\left(\frac{2 \delta_{\mathrm{SD}}}{r^{2}}\right)\left(1+\frac{\delta_{\mathrm{SD}}}{r}+\frac{1}{3}\left(\frac{\delta_{\mathrm{SD}}}{r}\right)^{2}\right)}{1+2 \frac{\delta_{\mathrm{SD}}}{r}\left(1+\frac{\delta_{\mathrm{SD}}}{r}+\frac{1}{3}\left(\frac{\delta_{\mathrm{SD}}}{r}\right)^{2}\right)}
$$

where $\gamma_{\mathrm{SD}}$ and $\delta_{\mathrm{SD}}$ are the surface tension and the Tolman length for spherical droplets. By assuming that $\delta_{\mathrm{SD}}$ is constant and neglecting the terms $\frac{\delta_{\mathrm{SD}}}{r}$ and $\left(\frac{\delta_{\mathrm{SD}}}{r}\right)^{2}$, a simplified Eq. (2) was obtained by integrating Eq. (12). Following this method, similar equations can be deduced for the cylindrical nanodroplet and nanobubble interfaces. Based on the definition of the Tolman length in this study, the equation for the cylindrical nanodroplet interface is

$$
\frac{1}{\gamma_{\mathrm{D}}} \frac{d \gamma_{\mathrm{D}}}{d r}=\frac{\left(\frac{\delta_{\mathrm{D}}}{r^{2}}\right)\left(1+\frac{1}{2} \frac{\delta_{\mathrm{D}}}{r}\right)}{1+\frac{\delta_{\mathrm{D}}}{r}\left(1+\frac{1}{2} \frac{\delta_{\mathrm{D}}}{r}\right)}
$$

where $\gamma_{D}$ is the surface tension for the cylindrical droplets in this study. The equation for the cylindrical nanobubble interface is

$$
\frac{1}{\gamma_{\mathrm{B}}} \frac{d \gamma_{\mathrm{B}}}{d r}=\frac{-\left(\frac{\delta_{\mathrm{B}}}{r^{2}}\right)\left(1-\frac{1}{2} \frac{\delta_{\mathrm{B}}}{r}\right)}{1-\frac{\delta_{\mathrm{B}}}{r}\left(1-\frac{1}{2} \frac{\delta_{\mathrm{B}}}{r}\right)}
$$


where $\gamma_{\mathrm{B}}$ is the surface tension for the cylindrical bubbles in this study. Adopting the same assumptions which Tolman used, Eqs. (13) and (14) can be integrated from the flat interface to a radius of interest $r=R_{S}$ respectively, giving the final expressions:

$$
\begin{aligned}
& \frac{\gamma_{\mathrm{D} R_{S}}}{\gamma_{\mathrm{F}}}=\frac{1}{1+\frac{\delta_{\mathrm{D}}}{R_{S}}} \\
& \frac{\gamma_{\mathrm{B} R_{S}}}{\gamma_{\mathrm{F}}}=\frac{1}{1-\frac{\delta_{\mathrm{B}}}{R_{S}}}
\end{aligned}
$$

\section{B. Simulation setup}

The surface tension of flat, cylindrical nanodroplet and nanobubble interfaces were simulated with classical MD simulations using the open-source code LAMMPS (the large-scale atomic/molecular massively parallel simulator). ${ }^{30}$ All length units given in this work are presented in reduced LennardJones (LJ) units where not explicitly specified. Two types of molecules were used in the flat interface cases: one type of gas molecules $(\mathrm{G})$ and one type of liquid molecules (L), with properties given in Table I. A liquid slab was placed in the middle of the simulation domain which has a size of $30 \times 30 \times 60$ (see Fig. 1). Periodic boundary conditions were applied in all three directions. The molecules interact with each other through a truncated LJ potential with a cut off chosen to be 5 sigma:

$$
U_{i j}(l)=4 \epsilon_{i j}\left[\left(\frac{\sigma_{i j}}{r_{i j}}\right)^{12}-\left(\frac{\sigma_{i j}}{r_{i j}}\right)^{6}\right]
$$

where $U_{i j}(l)$ and $r_{i j}$ are the potential and distance between molecule $i$ and $j . \epsilon_{i j}$ and $\sigma_{i j}$ are the interacting strength and the characteristic length between molecule $i$ and $j$.

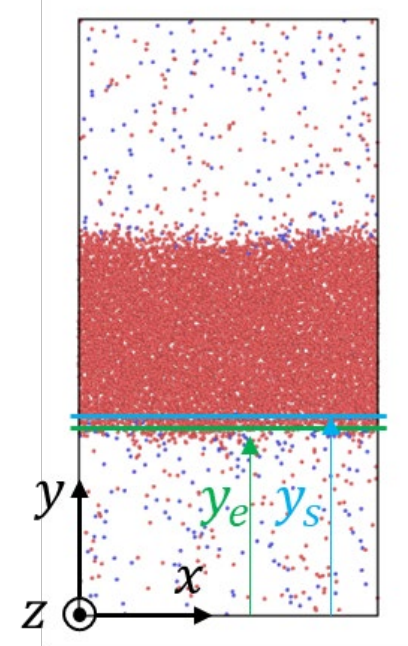

FIG. 1. Typical simulation box showing the coordinate system, equimolar surface, and surface of tension schematically for the flat interface case. $y_{e}$ and $y_{s}$ are the location of the equimolar surface and surface of tension, respectively. Red particles are the liquid molecules (L), blue particles are the gas molecules (G). 
In addition to the gas and liquid molecular types, three more molecule types are introduced in the nanodroplet and nanobubble interface cases: two types of solid molecules (hydrophilic $\left(\mathrm{S}_{\mathrm{phi}}\right)$ and hydrophobic $\left.\left(\mathrm{S}_{\mathrm{pho}}\right)\right)$ and one type of tether site molecules $\left(\mathrm{T}_{\mathrm{s}}\right)$. These are given in Table I. The G, $\mathrm{L}$, $\mathrm{S}_{\mathrm{phi}}$ and $\mathrm{S}_{\mathrm{pho}}$ molecules in the nanodroplet and nanobubble cases interact with each other through truncated LJ potentials (Formula 17) with a cut-off of 5 sigma.

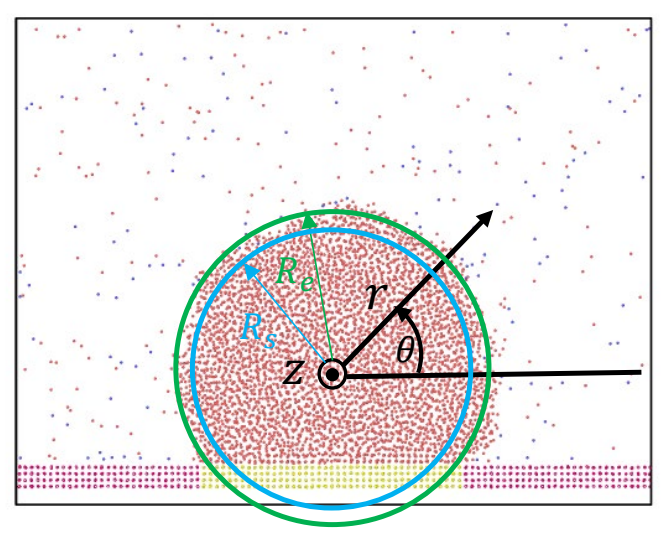

(a)

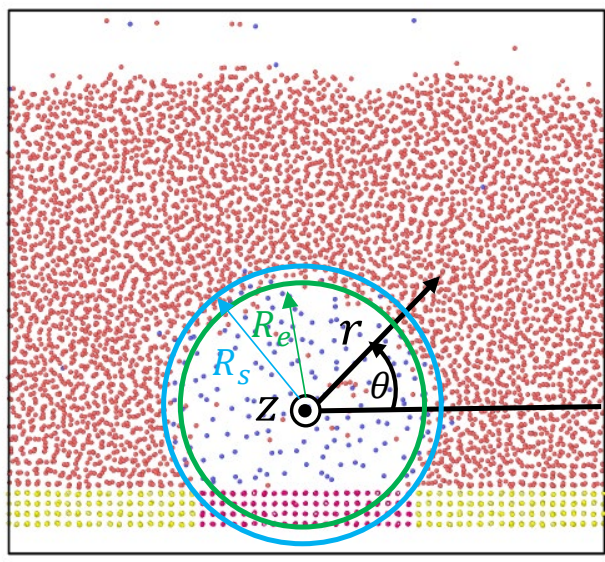

(b)

FIG. 2. Typical simulation boxes showing the coordinate system, equimolar surface and surface of tension schematically. (a) and (b) are nanodroplet and nanobubble cases, respectively. $R_{e}$ and $R_{s}$ are the curvature radii of the equimolar surface and the surface of tension. Red particles are the liquid molecules (L), blue particles are the gas molecules $(G)$, yellow particles are the hydrophilic wall molecules $\left(\mathrm{S}_{\mathrm{phi}}\right)$ and pink particles are the hydrophobic molecules $\left(\mathrm{S}_{\mathrm{pho}}\right)$. The nanodroplet and nanobubble have a cylindrical shape and the $\mathrm{z}$ axis is along the length of the cylinder.

The $\mathrm{T}_{\mathrm{s}}$ molecules are fixed in a $f c c$ lattice at the wall region with its density being 0.8 during the whole simulation. The $S_{\text {phi }}$ and $S_{\text {pho }}$ molecules are tethered to the $T_{s}$ molecules one by one through the following potential with a cut off of 5 sigma:

$$
U_{i j}(l)=\epsilon_{i j}\left(r_{i j}-r_{i 0}\right)^{2}
$$

where $\epsilon_{i j}=150$ is the interaction strength ${ }^{31}$ and $r_{i 0}=0$ is the equilibrium bond distance. Thus, the $\mathrm{S}_{\mathrm{phi}}$ and $\mathrm{S}_{\mathrm{pho}}$ molecules can vibrate around their tethering sites $\mathrm{T}_{\mathrm{s}}$.

The nanodroplets and nanobubbles have a cylindrical shape (see Fig. 2). Periodic boundary conditions were employed in the $x$ and $z$ directions. The top of the simulation domain has a wall boundary condition which interacts with $\mathrm{G}$ and $\mathrm{L}$ molecules through a soft potential to model an extended liquid region beyond the domain:

$$
U\left(r_{y i}\right)=\epsilon\left[\frac{2}{15}\left(\frac{\sigma}{r_{y i}}\right)^{9}-\left(\frac{\sigma}{r_{y i}}\right)^{3}\right]
$$


applied to molecules within a distance 2.5 from the wall. Here $\epsilon=1.0$ and $\sigma=1.0$ are the interacting strength and the characteristic length between the wall and molecules. $r_{y i}$ is the distance between molecule $i$ and the location of the wall. The thickness of the simulation domain in the $z$ direction is set to 5 , making the simulations effectively two dimensional.

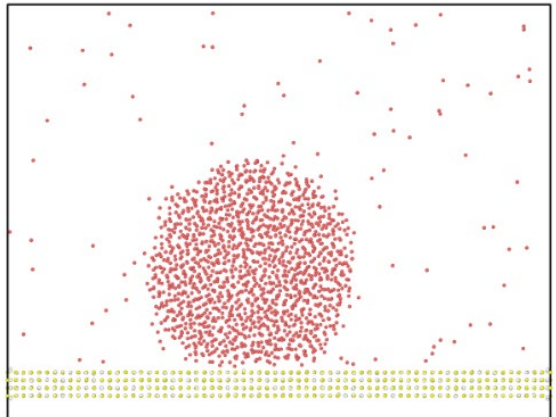

(a)

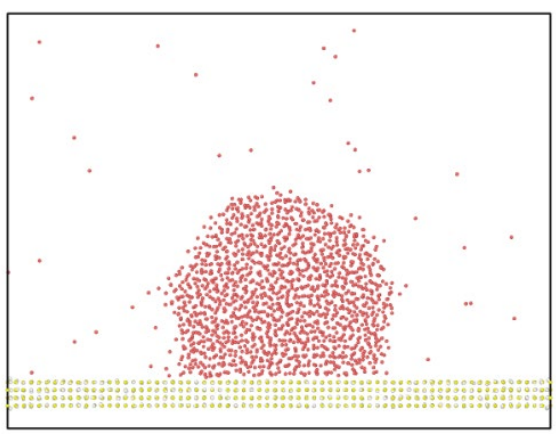

(c)

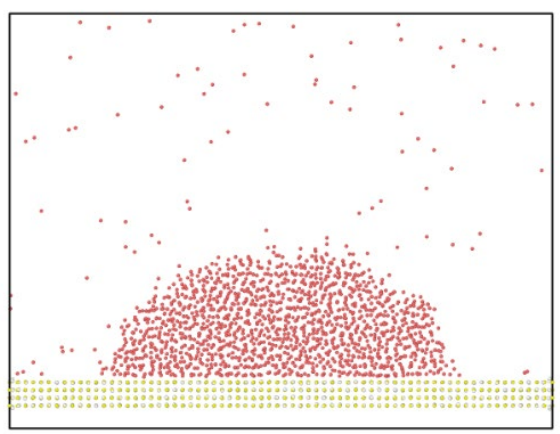

(e)

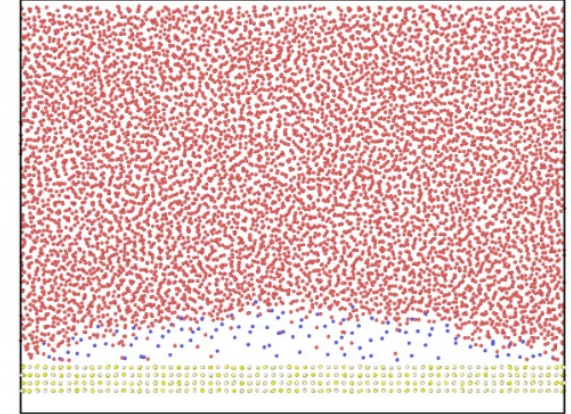

(b)

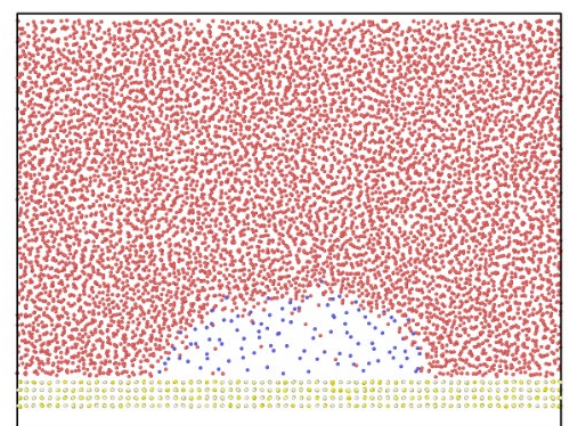

(d)

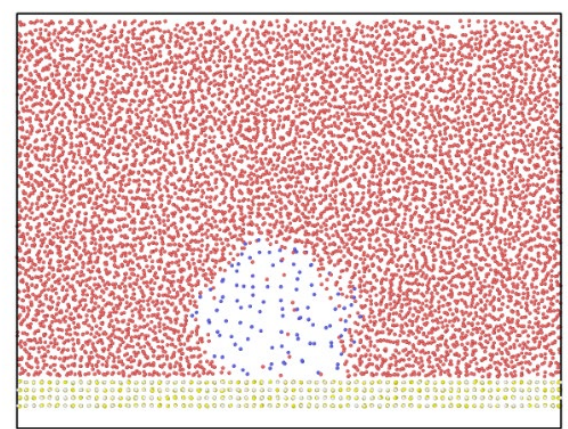

(f)

FIG. 3. Snapshots of droplets and bubbles on a homogeneous solid surface with $\epsilon_{\mathrm{SL}}$ being 0.2 for (a) and (b), 0.4 for (c) and (d) and 0.7 for (e) and (f).

For curved interface cases, there is a hydrophilic (hydrophobic) strip in the middle of the wall while the other part of the wall is hydrophobic (hydrophilic) for the nanodroplet (nanobubble) cases to control the size and anchor the position of the droplet (bubble). As a starting point, values of the interactions were chosen based on relevant values from Ref. 32. This was followed by a preliminary study to determine the parameters that provide stability for the simulations in terms of bubble/droplet size and interactions between liquid, gas and solid wall. As mentioned above, this was essential to overcome the potential limitations associated with the stable formation of nanobubbles reported in the literature. 
Particular attention was placed on the parameters for tuning the behaviour of the surface/fluid interactions (hydrophilic vs. hydrophobic). The hydrophobicity of the solid walls is controlled by tuning the interacting strength between solid and liquid molecules $\left(\epsilon_{\mathrm{Sphi}_{\mathrm{L}} \mathrm{L}}\right.$ and $\left.\epsilon_{\mathrm{S}_{\mathrm{pho}} \mathrm{L}}\right)$. The interacting strength between gas and wall molecules $\left(\epsilon_{\mathrm{S}_{\mathrm{phi}} \mathrm{G}}\right.$ and $\left.\epsilon_{\mathrm{Spho}_{\mathrm{p}}}\right)$ were also controlled to obtain aerophilic and aerophobic properties on the hydrophobic and hydrophilic wall surfaces, respectively. This helps to anchor the droplet (bubble) on the hydrophilic (aerophilic) area. The interacting strength and length between gas molecules were increased to form a thin but at the same time stable gas phase. Examples of the investigation on the effect of $\epsilon_{\mathrm{Sphi}_{\mathrm{i}} \mathrm{L}}$ and $\epsilon_{\mathrm{S}_{\mathrm{pho}} \mathrm{L}}$ on the wettability of the solid wall are shown in Fig. 3. The interacting strength and characteristic length between different molecules are the same except $\epsilon_{\mathrm{SL}}$, which is set to 0.2 for (a) and (b), 0.4 for (c) and (d) and 0.7 for (e) and (f). Each image is a snapshot of the simulation when the system is balanced. It is shown that the liquid contact angle of the droplet on the solid wall decreases as $\epsilon_{\mathrm{SL}}$ increases while the air contact angle of the bubble increases. Following this preliminary investigation, the parameters set in this study are chosen as shown in Table I.

TABLE I. Values of LJ potential parameters used in the MD simulations

\begin{tabular}{ccc}
\hline \hline$i-j$ & $\epsilon_{i j}$ & $\sigma_{i j}$ \\
\hline $\mathrm{L}-\mathrm{L}$ & 1.0 & 1.0 \\
$\mathrm{~S}_{\mathrm{phi}}-\mathrm{L}$ & 1.0 & 1.0 \\
$\mathrm{~S}_{\mathrm{pho}}-\mathrm{L}$ & 0.1 & 1.0 \\
$\mathrm{~L}-\mathrm{G}$ & 0.52 & 1.18 \\
$\mathrm{~S}_{\mathrm{phi}}-\mathrm{G}$ & 0.1 & 1.18 \\
$\mathrm{~S}_{\mathrm{pho}}-\mathrm{G}$ & 0.5 & 1.18 \\
$\mathrm{G}-\mathrm{G}$ & 0.5 & 1.7 \\
\hline \hline
\end{tabular}

\section{Simulation procedure}

All the simulations were performed in a three-dimensional Cartesian coordinate system. The time step was set at $\Delta \mathrm{t}=\sigma_{\mathrm{LL}} \sqrt{\left(m / \epsilon_{L L}\right)} / 500=0.002$, where $\mathrm{m}=1$ is the mass of the liquid molecules. It is sufficiently smaller than the shortest time scale available in the system. For the flat interface cases, initially, regions of gas and liquid molecules are added to the simulation domain. The thickness of the liquid slab is around 20. First, the system is equilibrated in an NVT ensemble at a temperature of $T_{\text {set }}=$ 0.8 using a Nose-Hoover thermostat with a heat bath size of $\mathrm{Q}=0.2$ for 3 million time steps. Measurements of the density and pressure tensor are then obtained using a grid of $0.5 \times 0.5$ chunks covering the $x-y$ plane in an NVE ensemble run over 500,000 time steps. The time-average values are calculated by taking a sample every 50 time steps. As the flat gas/liquid interface is parallel to the 
$x-y$ plane, the normal pressure component is $P_{\mathrm{N}}=P_{y y}$ and the tangential pressure component is $P_{\mathrm{T}}=\left(P_{x x}+P_{z z}\right) / 2$.

For the nanodroplet and nanobubble cases, initially, gas and liquid molecules were placed on the hydrophobic and hydrophilic surfaces, respectively. Then the system was equilibrated for 3 million time steps in an NVT ensemble using a Nose-Hoover thermostat with the temperature of the wall molecules $\left(\mathrm{S}_{\mathrm{phi}}\right.$ and $\left.\mathrm{S}_{\mathrm{pho}}\right)$ thermostatted at a temperature of $T_{\text {set }}=0.8$. The heat bath size was $\mathrm{Q}=0.2$. When the system is equilibrated, gas molecules tend to stay on the hydrophobic surfaces and the liquid molecules preferentially stay on the hydrophilic surfaces. In this way, we were able to form cylindrical nanodroplets and nanobubbles on solid surfaces. Data collection follows the same process as the flatinterface case. Time-averaged density and pressure tensor measurements were obtained in a grid of chunks with the size being $0.5 \times 0.5$ covering the $x-y$ plane in an NVE ensemble run over 500,000 time steps by taking a sample every 50 time steps. After that, a cylindrical coordinate system was established by fitting a circle to the location halfway between bulk liquid and bulk gas density. Then the pressure tensors in the three-dimensional Cartesian coordinate system were transferred to the cylindrical coordinate system (Fig. 4) using the following equations:

$$
\begin{gathered}
P_{\mathrm{N}}=P_{x x}(\cos (\theta))^{2}+P_{y y}(\sin (\theta))^{2}+P_{x y} \sin (2 \theta) \\
P_{\mathrm{T}}=P_{x x}\left(\cos \left(\theta+\frac{\pi}{2}\right)\right)^{2}+P_{y y}\left(\sin \left(\theta+\frac{\pi}{2}\right)\right)^{2}+P_{x y} \sin (2 \theta+\pi)
\end{gathered}
$$

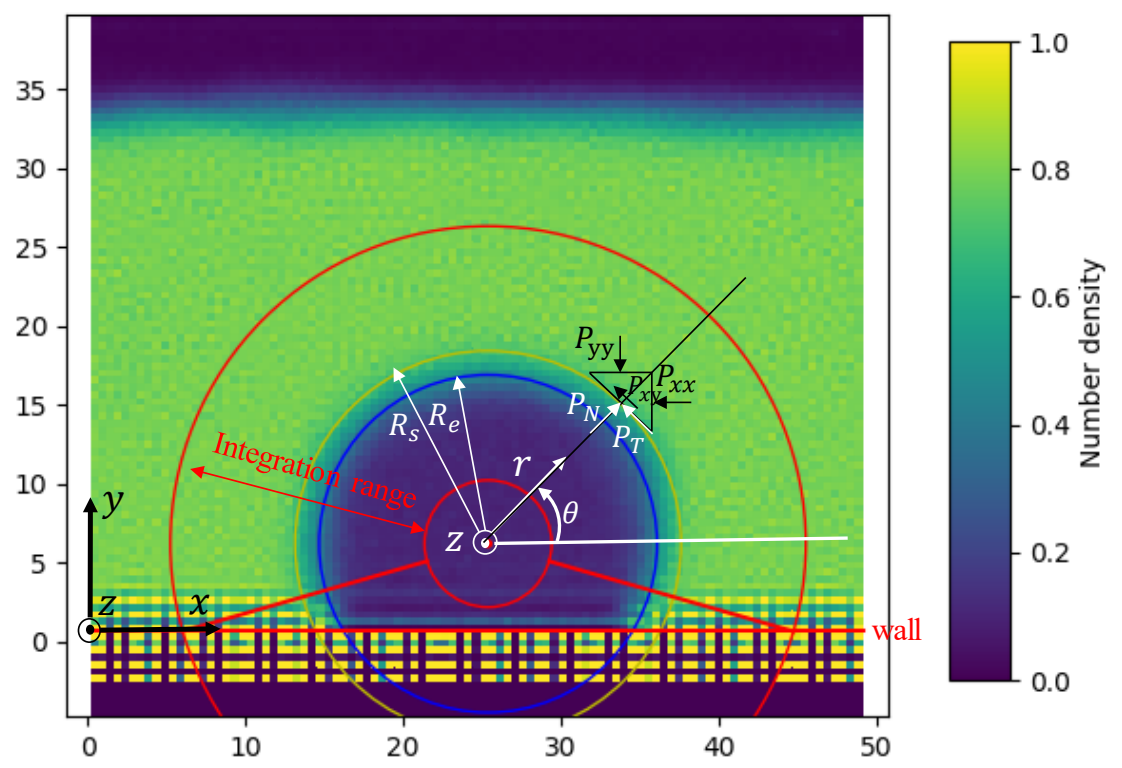

FIG. 4. A typical density profile of a nanobubble case. The three-dimensional Cartesian coordinate system at the down-left corner was used in the MD simulation. The cylindrical coordinate system was established by fitting the equal-density profile. The $z$ axis is along the length of the cylindrical bubble. In the $r$ direction, the integration of the surface tension starts from the bulk gas region inside the bubble (the inner red circle) and ends at the bulk liquid region (the outer red circle). 


\section{RESULTS AND DISCUSSION}

\section{A. The 'average after' procedure of calculating the surface tension and Tolman length}

To calculate the surface tension $(\gamma)$ and the Tolman length $(\delta)$ with the density $(\rho)$, normal $\left(P_{\mathrm{N}}\right)$ and tangential $\left(P_{\mathrm{T}}\right)$ pressures, there are two procedures available. Procedure i) (referred to as 'average after') is to get the local profiles of $\rho, P_{\mathrm{N}}$, and $P_{\mathrm{T}}$ in the normal direction ( $y$ and $r$ directions for the flat and curved interface cases, respectively). These profiles are then used to calculate the local $\gamma$ and $\delta$ in the whole range of the tangential direction ( $x$ and $\theta$ directions for the flat and curved interface cases, respectively). Finally, the mean values of $\gamma$ and $\delta$ can be obtained by averaging the local values along the tangential direction. Procedure ii) (referred to as 'average before') is to obtain the mean profiles of $\rho, P_{\mathrm{N}}$, and $P_{\mathrm{T}}$ in the normal direction first by averaging the local profiles along the tangential direction. Then, the mean value of $\gamma$ and $\delta$ can be calculated using the mean profiles of $\rho, P_{\mathrm{N}}$, and $P_{\mathrm{T}}$.

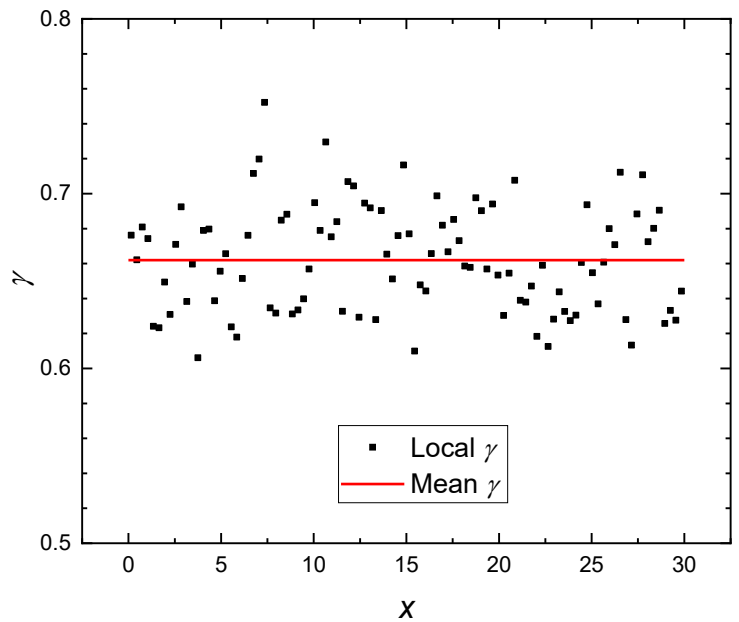

(a)

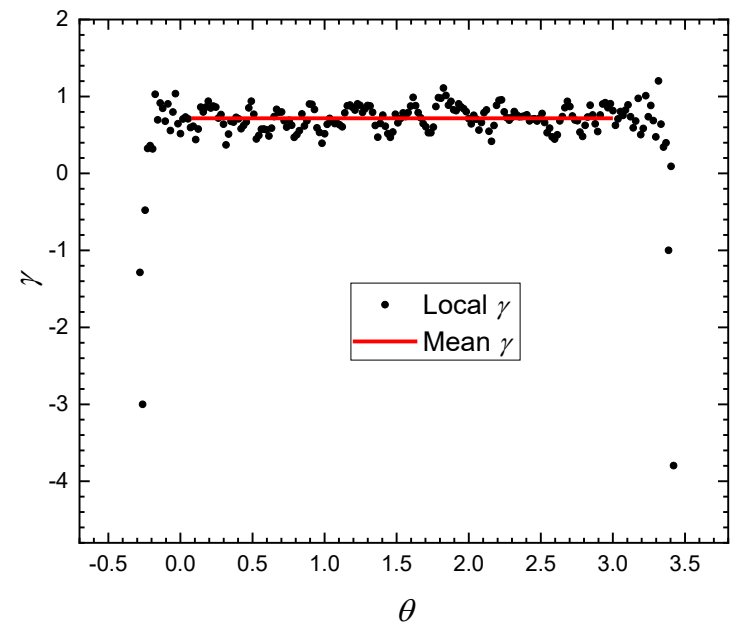

(c)

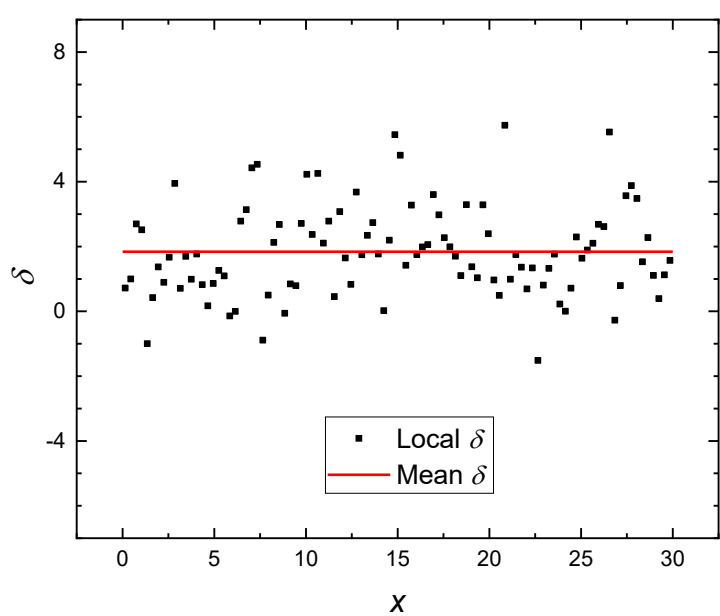

(b)

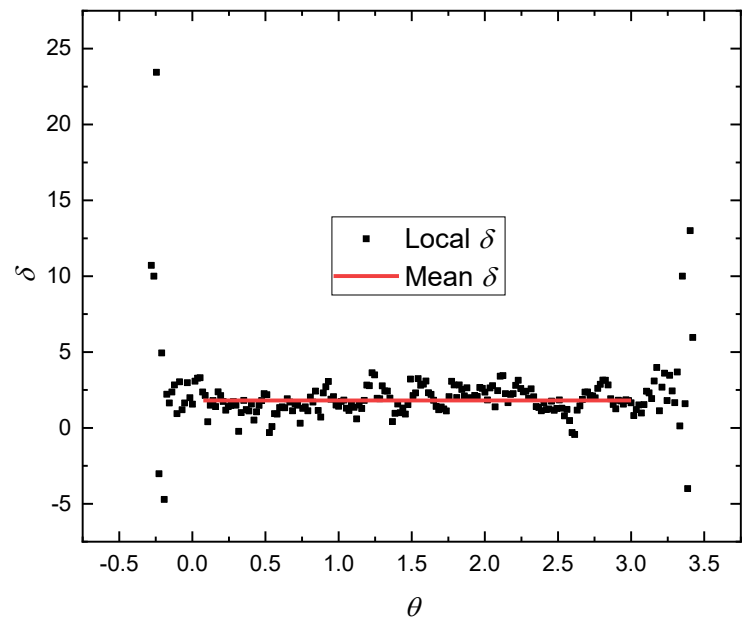

(d)

FIG. 5. (a) Surface tension, and (b) Tolman length along a flat liquid/gas interface. (c) Surface tension and (d) Tolman length along the liquid/gas interface of a nanobubble case. 
Figure 5 (a) and (b) show the local and mean values of $\gamma$ and $\delta$ along a flat gas/liquid interface. The mean $\gamma$ and $\delta$ are obtained by averaging the local values along the whole range in the $x$ direction which is parallel to the interface. It can be seen that the local $\gamma$ slightly fluctuates while the data points are evenly scattered around the mean value. Because periodic boundary conditions are applied in all directions, the lateral boundaries do not show any effect on the local surface tension. While the fluctuation of the local $\delta$ is relatively high compared to the mean value. But the data points are also evenly scattered around the mean value.

Distributions of the local $\gamma$ and $\delta$ in the $\theta$ direction obtained with the local $\rho, P_{\mathrm{N}}$ and $P_{\mathrm{T}}$ profiles for a nanobubble case are shown in Fig. 5 (c) and (d). We can see that the solid wall has a strong effect on $\gamma$ and $\delta$ near the wall region. As the focus of this study is on the liquid/gas interface, the mean surface tension in the 'average after' method is obtained by averaging the value in the middle flat area which is far from the wall region. It is shown that the distribution of the local $\gamma$ and $\delta$ as a function of $\theta$ for the nanobubble interface far from the wall region is similar to that for the flat interface case.

\section{B. The 'average before' procedure of calculating the surface tension and Tolman length}

The 'average before' procedure of calculating the surface tension is shown in this section. In this procedure, the profiles of the mean density, normal and tangential pressure components are obtained first by averaging the local profiles along the tangential direction ( $x$ and $\theta$ direction for the flat and curved interface, respectively). The surface tension is then calculated using the mean pressure component profiles.

Figure 6 shows examples of the mean density and pressure component profiles for both flat and curved interfaces. As already discussed, since the wall can have a strong effect on the density and pressure components for curved interface cases, the mean profiles are calculated using data from the range of $\theta$ far from the wall. For the flat interface cases, given the absence of solid walls, the mean profiles are obtained using data in the whole range of $x$.

For the flat interface (Fig. 6 (a) and (b)), the profiles start from the bulk gas region and ends in the bulk liquid region. For the droplet (Fig. 6 (c) and (d)) cases, the origin of the system is at the centre of the nanodroplet and the profile starts from inside the droplet and ends deep inside the bulk gas region. For the nanobubble (Fig. 6 (e) and (f)) cases, the origin of the system is at the centre of the nanobubble and the profile starts from inside the bubble and ends deep inside the bulk liquid region. 


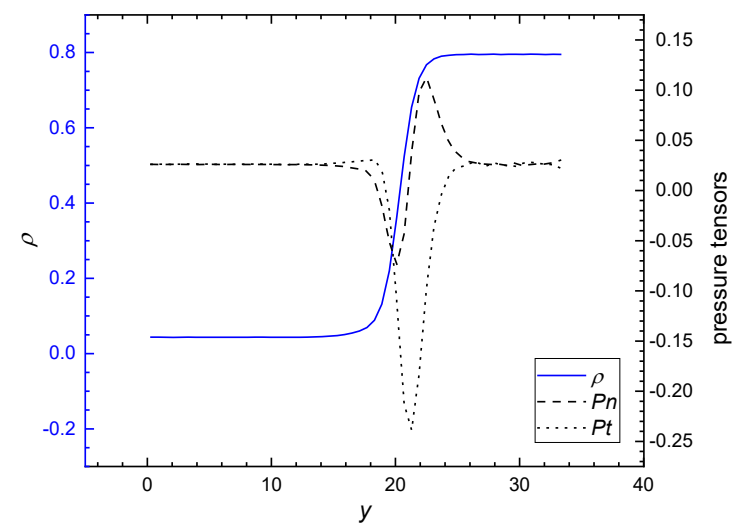

(a)

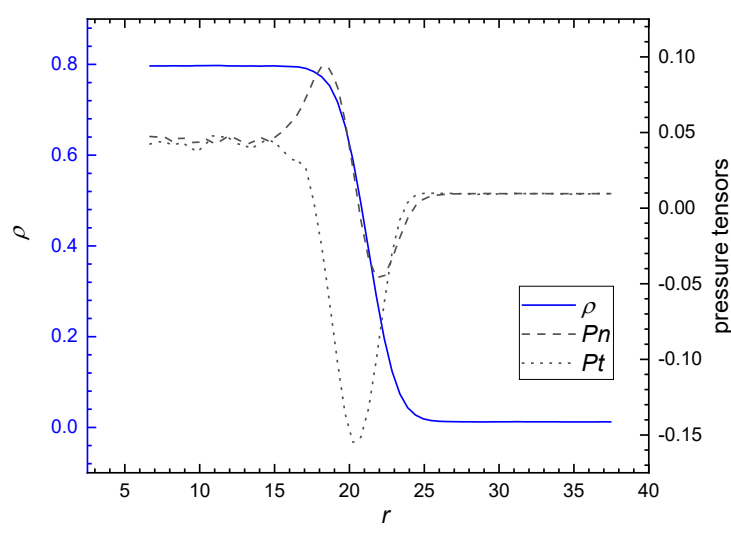

(c)

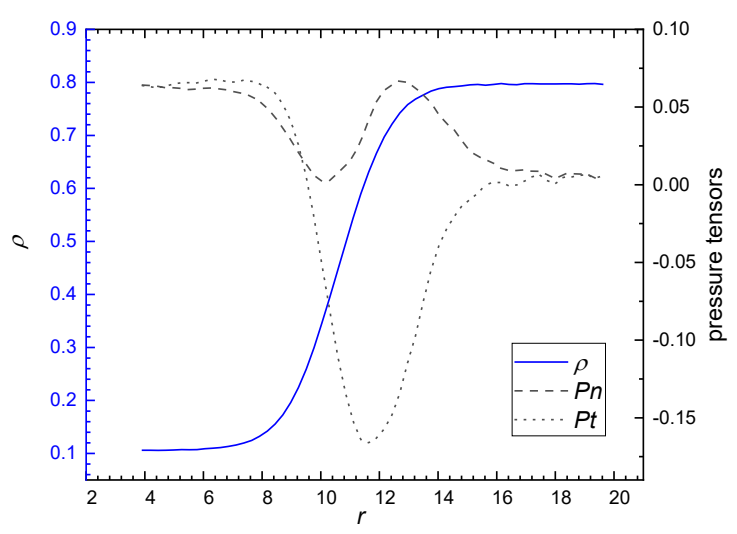

(e)

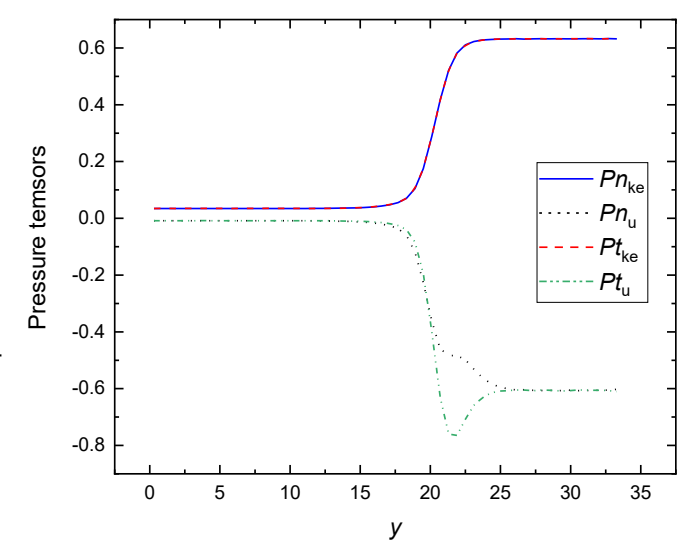

(b)

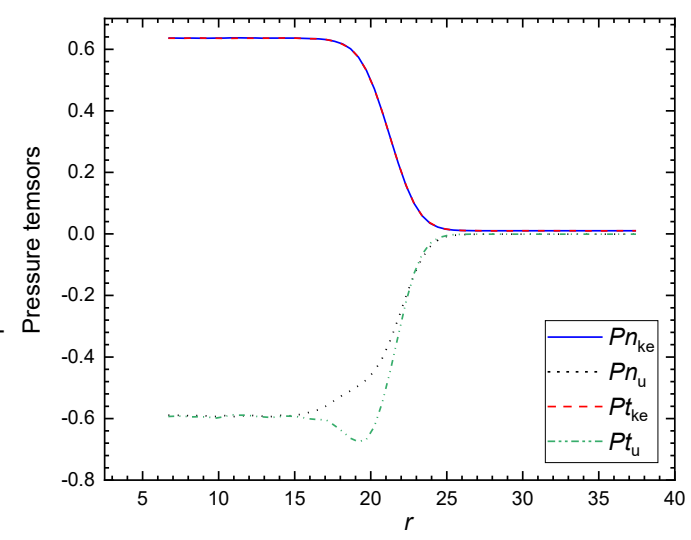

(d)

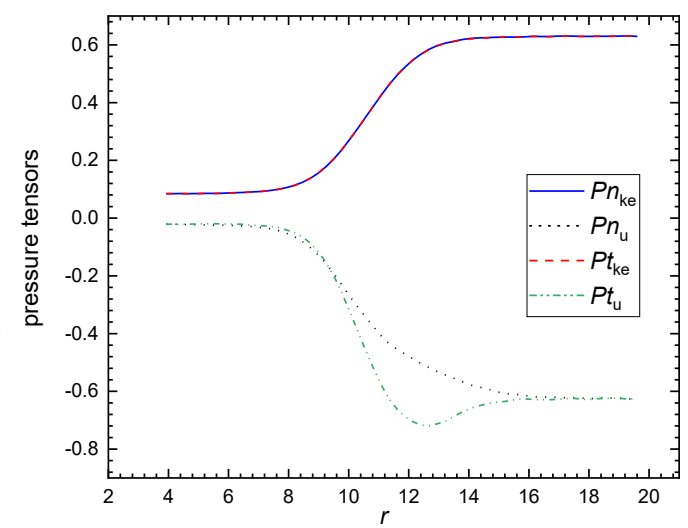

(f)

FIG. 6. Average density and pressure component profiles for both flat and curved interfaces. (a), (c) and (e) Density, normal and tangential pressure component profiles for flat, nanodroplet and nanobubble interfaces, respectively. (b), (d) and (f) Kinetic and virial components of the normal and tangential pressure for flat, nanodroplet and nanobubble interfaces, respectively.

We can see from Fig. 6 (a) that normal and tangential pressure components are the same in both bulk gas and liquid regions, which means there is no pressure difference across the flat interface. Near an interface in a balanced system, a constant normal pressure is required for momentum balance. While this is known to not occur for solid-liquid interfaces with the virial or IK1 pressure (note here that IK1 is the first term in the Irving Kirkwood expansion, which is the same as the virial from LAMMPS) ${ }^{33}$ 
and the default pressure measured by LAMMPS. This observation motivates the use of the VA and MOP formulations for liquid/solid studies ${ }^{34,35}$. Calculation of these pressures is complicated ${ }^{36}$, especially for a curved interface. In this work, the IK1 pressure is used for simplicity, as the normal pressure does not affect the resulting surface tension (the integration of the two opposite peaks in the normal pressure component with respect to radius is zero). The Tolman length is known to be affected by choice of contour (see e.g. Ref. 25), but the presented trends compare bubbles and droplets calculated in a consistent manner; as there is no unique form, we use the simplest, leaving a more detailed study of the a full interface tracking pressure to future work. Thus, for all the interfaces, the normal pressure component (Fig. 6 (a), (c) and (e)) shows a higher peak on the liquid side and a lower peak on the gas side. The tangential pressure component instead is shown to have a single deep valley. It is this difference between the normal and tangential pressure components which is a direct manifestation and can be used to quantify the surface tension.

Figure 6 (b) (d) and (f) show the kinetic and virial parts of the normal and tangential pressure components. It can be seen that no matter if the interface is curved or not, the magnitude of both the kinetic and virial parts of the normal and tangential pressure components increase monotonically from the gas region to the liquid region (except the virial part of the tangential pressure, which shows a peak on the liquid side near the interface). The kinetic part of the pressure is positive while the virial part is negative. When these kinetic and viral parts are added up, the total pressure follow the Young-Laplace law which predicts a higher total pressure on the side to which the interface is curved. It is also shown that the kinetic parts of the normal and tangential pressure components equal each other for all interfaces which means that the kinetic part of the pressure does not contribute to the surface tension. The difference between the virial parts of the normal and tangential pressure components near the interface is the origin of the surface tension.

\section{The surface tension and Tolman length}

The errors for all the results in this work are from time-average values of four subsequent subsets of 500,000 time steps. The $\gamma$ and $\delta$ calculated using the two different procedures are compared. The results show that the $\gamma$ and $\delta$ of the flat interface calculated using the two procedures are identical while those for curved interfaces are slightly different. Examples of the $\gamma$ and $\delta$ for nanodroplet interfaces calculated with two procedures are shown in Fig. B.1. The differences of $\gamma$ calculated by the two procedures are small while the differences in $\delta$ increases slightly as the curvature radius of the nanodroplet increases. The results discussed later in this study are all calculated using the 'average before' procedure ("average before" chosen arbitrarily given no clear advantage of either, but not expected to affect conclusions). 

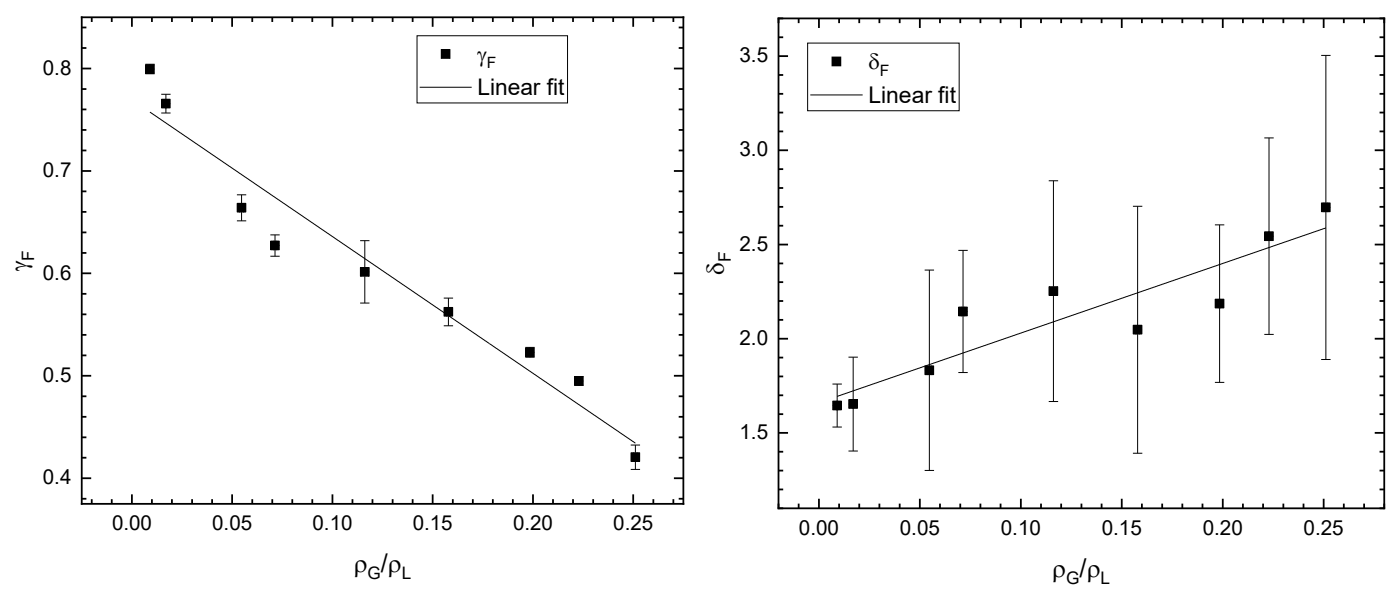

FIG. 7. Surface tension depicted as $\gamma_{\mathrm{F}}$ and Tolman length depicted as $\delta_{\mathrm{F}}$ for flat interfaces with different $\rho_{\mathrm{G}} / \rho_{\mathrm{L}}$.

The effect of $\rho_{\mathrm{G}} / \rho_{\mathrm{L}}$ on $\gamma$ and $\delta$ is studied with flat interface cases and the results are shown in Fig. 7, where we change $\rho_{\mathrm{G}} / \rho_{\mathrm{L}}$ by change the number of gas molecules in the gas region of the system, which has a fixed volume. It should be noted here that the density used in this study is the particle number density. The density is directly related to pressure at the same temperature. The smallest $\rho_{\mathrm{G}} / \rho_{\mathrm{L}}$ we can get is the case where there are no gas molecules in the gas region, with the gas region filled by the liquid vapour molecules. The highest $\rho_{\mathrm{G}} / \rho_{\mathrm{L}}$ we can get is around 0.25 , above which the boundary between the gas and liquid is hard to identify. It should be noted that in the whole density ratio range, the density of the liquid region is quite stable and stays around 0.8. That is because liquid is less compressible than gas. We can see from Fig. 7 (a) that $\gamma$ decreases as $\rho_{\mathrm{G}} / \rho_{\mathrm{L}}$ increases (or as the density difference between gas and liquid decreases).

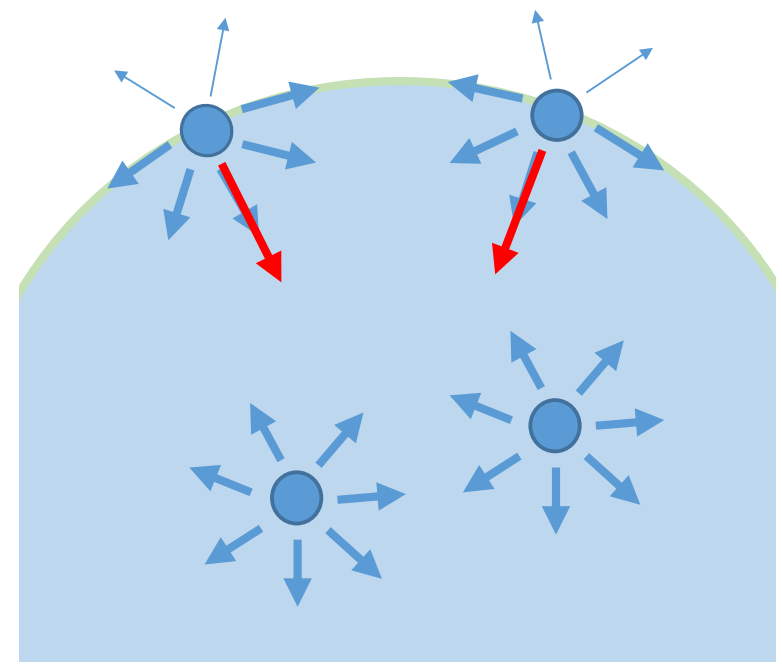

FIG. 8. Schematic diagram showing typical force state of liquid molecules.

The reason for this decrease is that $\gamma$ results from the unbalanced intermolecular forces at the interface between the gas and liquid regions (as shown schematically in Fig. 8). The molecules on the interface experience a resultant force pointing into the liquid which means it tends to contract. As the liquid 
density is relatively constant, when there are fewer gas molecules in the gas region, they contribute less force to the interface which results in an unbalanced force state at the interface and a higher surface tension. When the gas density increases, the unbalanced force state of the interface eases and the surface tension decreases.

It is shown in Fig. 7 (a) that the surface tension of a flat interface $\left(\gamma_{\mathrm{F}}\right)$ has an approximately linear relationship with $\rho_{\mathrm{G}} / \rho_{\mathrm{L}}$, which is in agreement with results from other researches where the surface tension decreases linearly with the supersaturation ratio ${ }^{37}$. Thus, a linear fitting is applied to the data:

$$
\gamma_{\mathrm{F}}=-1.3352 \frac{\rho_{\mathrm{G}}}{\rho_{\mathrm{L}}}+0.7695
$$

Moreover, $\rho_{\mathrm{G}} / \rho_{\mathrm{L}}$ also shows an effect on the Tolman length (see Fig. 7 (b)). The Tolman length ranges from 1.2 to 3.5 reduced units. Similar to the results of surface tension, a linear relationship is used to fit the increase of the Tolman length with $\rho_{\mathrm{G}} / \rho_{\mathrm{L}}$ :

$$
\delta_{\mathrm{F}}=3.6941 \frac{\rho_{\mathrm{G}}}{\rho_{\mathrm{L}}}+1.6605
$$

where $\delta_{\mathrm{F}}$ represents the Tolman length for a flat interface. It should be noted here that as the relative uncertainty of $\delta_{\mathrm{F}}$ is high, the linear fit can have a big variation.

These linear fits will be used to subtract $\gamma_{\mathrm{F}}$ and $\delta_{\mathrm{F}}$ (with the same $\rho_{\mathrm{G}} / \rho_{\mathrm{L}}$ as the curved interfaces) from curved interfaces to obtain the effect of curvature.

Molecular dynamics simulations necessarily contain a finite number of molecules, with periodic boundaries used at the edges. In order to ensure the finite-size of these systems do not affect the observed $\gamma$ and $\delta$, simulations have been performed to parameterise the effect of the simulation domain size. To demonstrate this, a set of simulations (referred to as 'larger simulation domain') which use twice as much space between the nanobubble/nanodroplet and the boundary as the remaining simulations were conducted. Results of these larger simulation domains are consistent with the smaller domains used in the majority of this work.
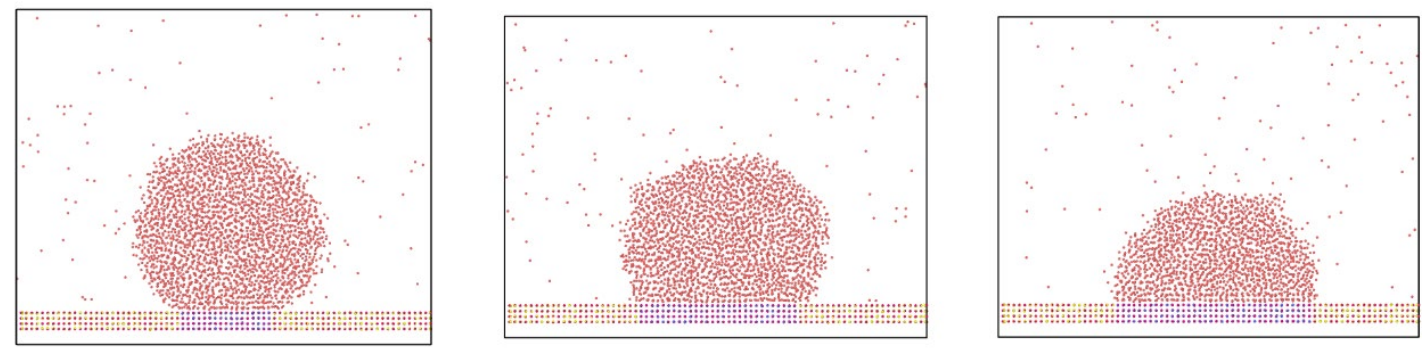

FIG. 9.Nanodroplets with close curvature radii but different intrusions into the simulation domain.

The effect of the intrusion of the curved interface into the simulation domain on $\gamma$ and $\delta$ has also been studied. Here, the intrusion of the curved interface is defined as distance between the fitted origin of the interface and the flat wall in the direction from wall to the curved interface normalised by the equimolar 
curvature radius of the curved interface. It is determined by the curvature radius of the curved interface and the length of the pinning pattern. By controlling the length of the pinning pattern and the number of the liquid molecules, droplets with similar curvature radii but different intrusion can be obtained (referred to as 'different intrusions' and examples of droplets with close curvature radius but different intrusions are shown in Fig. 9).

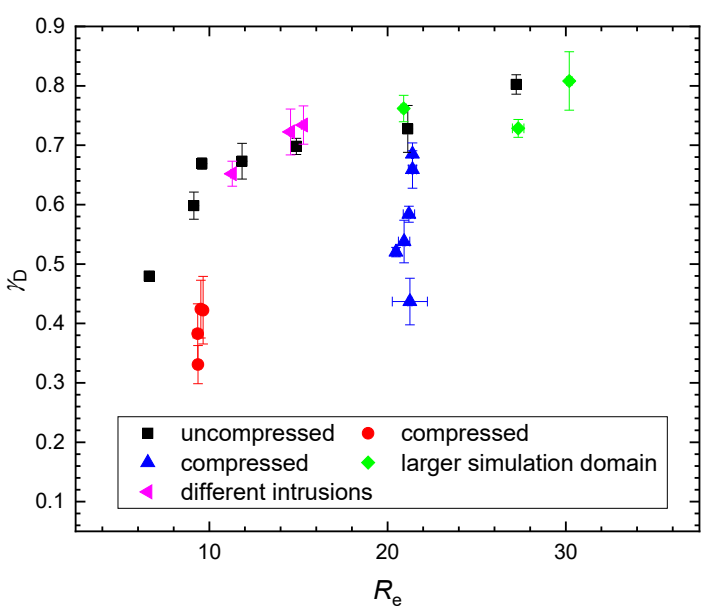

(a)

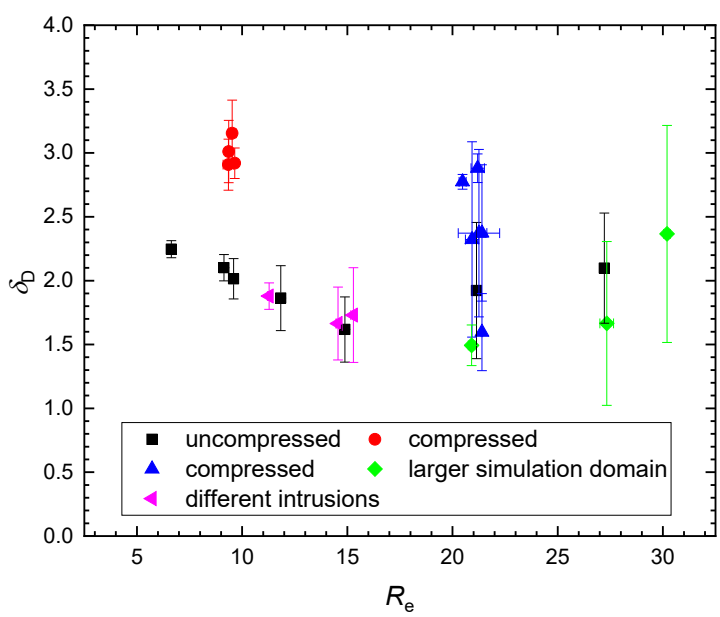

(c)

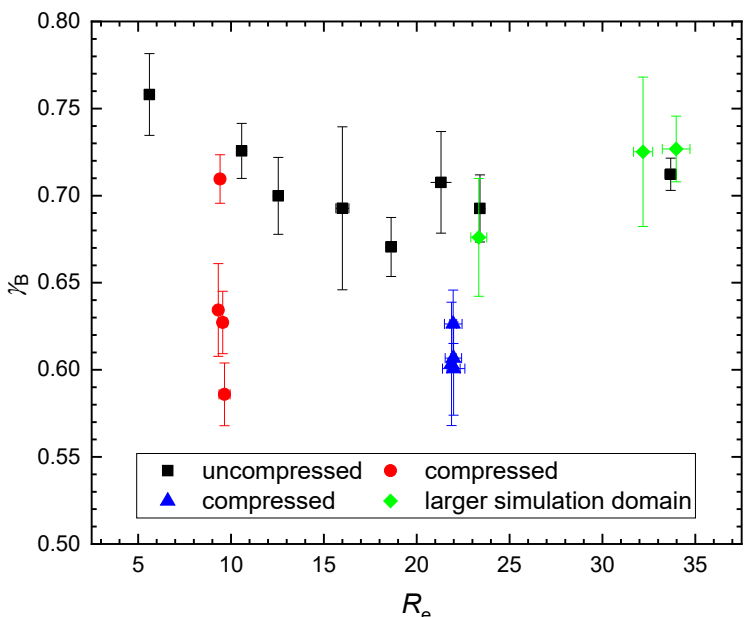

(b)

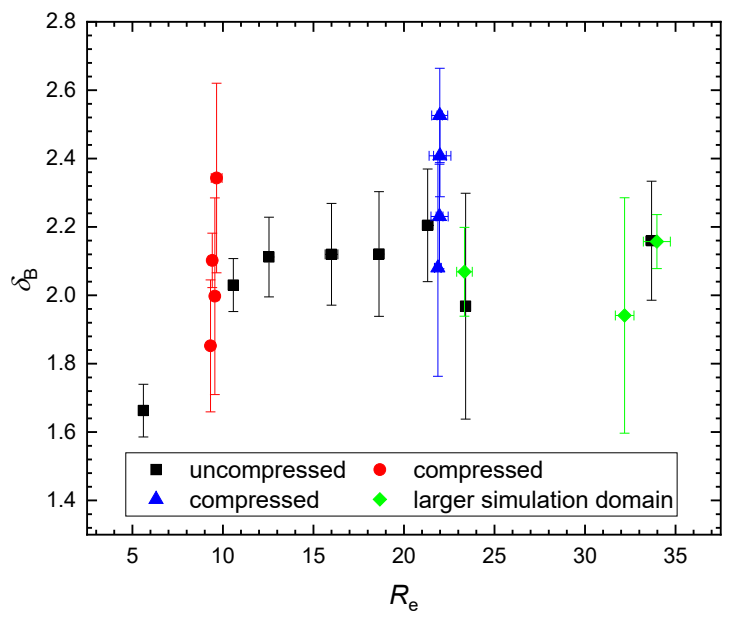

(d)

FIG. 10. The surface tension and Tolman length as a function of $R_{e}$. (a) and (b) Surface tension against $R_{e}$ for nanodroplets $\left(\gamma_{\mathrm{D}}\right)$ and nanobubbles $\left(\gamma_{\mathrm{B}}\right)$, respectively. (c) and (d) Tolman length against $R_{e}$ for nanodroplets $\left(\delta_{\mathrm{D}}\right)$ and nanobubble $\left(\delta_{\mathrm{B}}\right)$, respectively. This figure shows all curved interface cases run.

Figure 10 shows $\gamma$ and $\delta$ for all curved interface cases run in this work. Results labelled 'larger simulation domain' and 'different intrusions' show similar values of $\gamma$ and $\delta$ to the remaining results which confirms that the size of the simulation domain used in the remaining studies is sufficient to avoid finite size effects, and the intrusion of the nanodroplet interface does not affect the measured values of $\gamma$ and $\delta$. Figure 10 (a) and (b) show the surface tension of nanodroplet $\left(\gamma_{\mathrm{D}}\right)$ and nanobubble interfaces $\left(\gamma_{\mathrm{B}}\right)$ with different $R_{e}$ and $\rho_{\mathrm{G}} / \rho_{\mathrm{L}}$. In all simulations, nanodroplets stay on the solid surfaces and are surround by gas region (see Fig. 2 (a)). For the uncompressed nanodroplet cases (labelled as 'uncompressed'), there are no gas molecules added in the system, thus the pressure in the bulk gas 
region is the saturated liquid-vapour pressure. While the pressure inside the droplet is governed by the Yong-Laplace law. For the compressed nanodroplet cases (labelled as 'compressed'), different amounts of gas molecules are added in the bulk gas region to adjust the pressure of the system. All the nanobubbles stay on the solid surface and are submerged in liquid (see Fig. 2 (b)). For the uncompressed nanobubble cases (labelled as 'uncompressed'), there is a vapour region above the liquid region which ensures that the pressure in the bulk fluid equals the saturated liquid-vapour pressure. While the pressure inside the bubble is determined by the Young-Laplace law. For the compressed nanobubble cases (labelled as 'compressed'), there is no vapour region above the bulk liquid region, and the pressure of the system can be adjusted by the number of gas molecules added inside the bubble.

As we can see from Fig. 10 (a), $\gamma_{D}$ of uncompressed nanodroplets increases monotonically with the molecular curvature radius and levels off at a $R_{e}$ of around 20. Meanwhile, $\gamma_{\mathrm{D}}$ of compressed droplets is smaller than that of the uncompressed droplets with the same $R_{e}$ which is in accordance with the flat interface cases where $\gamma_{\mathrm{F}}$ decreases as $\rho_{\mathrm{G}} / \rho_{\mathrm{L}}$ increases. $\gamma_{\mathrm{B}}$ of the uncompressed nanobubble interface (shown in Fig. 10 (b)) decreases first and then increases slightly as $R_{e}$ increases. Similarly, $\gamma_{\mathrm{B}}$ of compressed bubbles is smaller than that of the uncompressed bubbles with the same $R_{e}$. When $R_{e}$ is smaller than $20, \gamma_{\mathrm{D}}$ shows an opposite trend compared to $\gamma_{\mathrm{B}}$, where $\gamma_{\mathrm{D}}$ decreases as $R_{e}$ decreases while $\gamma_{\mathrm{B}}$ increases.

The results for Tolman length of the nanodroplet $\left(\delta_{\mathrm{D}}\right)$ and nanobubble $\left(\delta_{\mathrm{B}}\right)$ interfaces are shown in Fig. 10 (c) and (d), respectively. For both nanobubble and nanodroplet results, $\delta$ for compressed cases is slightly higher than the uncompressed cases which is in accordance with the flat interface cases where higher $\rho_{\mathrm{G}} / \rho_{\mathrm{L}}$ leads to bigger $\delta_{\mathrm{F}}$. When $R_{e}$ is smaller than $20, \delta$ also shows opposite trends for the nanodroplet and nanobubble interfaces, where $\delta_{\mathrm{D}}$ increases as $R_{e}$ decreases while $\delta_{\mathrm{B}}$ decreases. When $R_{e}$ is larger than 20 , the $\delta_{\mathrm{D}}$ levels off smoothly while $\delta_{\mathrm{B}}$ shows a slight decrease and then levels off. The results show that $\gamma$ and $\delta$ for nanodroplet and nanobubble interfaces have different trends with the curvature radius. However, this is a combination of the effect of $\rho_{\mathrm{G}} / \rho_{\mathrm{L}}$ and curvature. In the following section, the effect of curvature is obtained and discussed.

\section{The effect of curvature on the surface tension and Tolman length}

As Fig. 7 (a) has shown, the effect of $\boldsymbol{\rho}_{\mathrm{G}} / \boldsymbol{\rho}_{\mathrm{L}}$ on $\boldsymbol{\gamma}_{\mathbf{F}}$ can be fitted to a linear relationship described with Eq. (22). Thus, the effect of the density ratio can be corrected in the droplet and bubble cases by using $\gamma_{\mathrm{F}}$ with the same $\rho_{\mathrm{G}} / \boldsymbol{\rho}_{\mathrm{L}}$. 


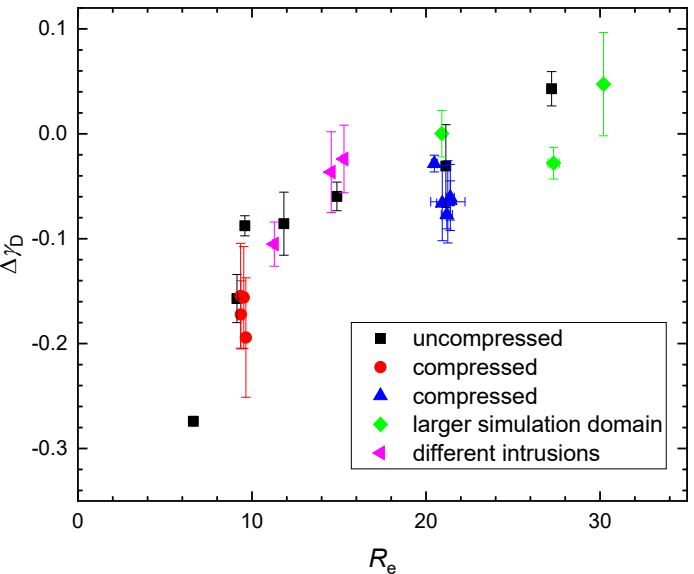

(a)

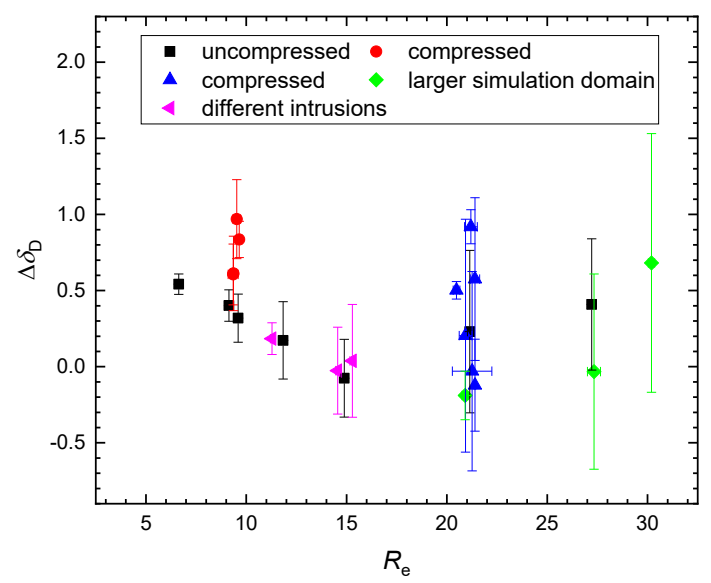

(c)

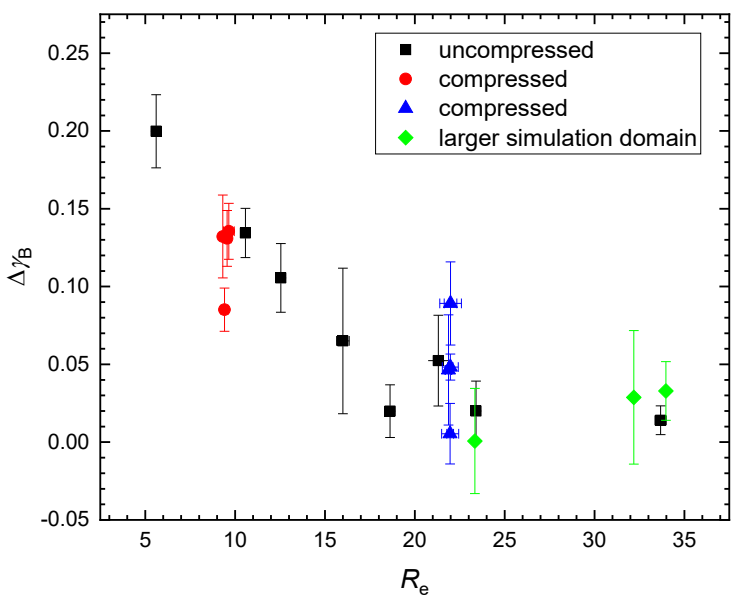

(b)

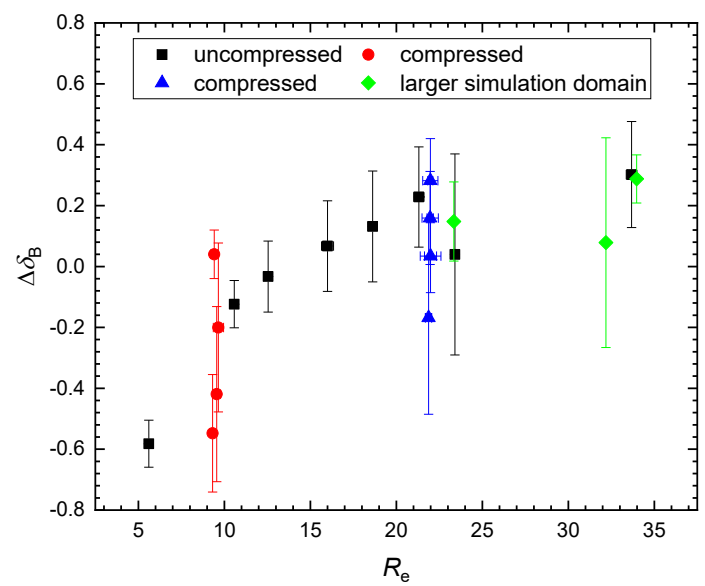

(d)

FIG. 11. Effect of curvature on $\gamma$ and $\delta$ obtained by subtracting the effect of $\rho_{\mathrm{G}} / \rho_{\mathrm{L}}$ from the results in Fig. 7. (a) and (b) Curvature dependence of $\gamma$ for nanodroplets and nanobubbles, respectively. (c) and (d) Curvature dependence of $\delta$ for nanodroplets and nanobubbles, respectively. This figure shows all cases run.

The difference between $\gamma_{\mathrm{F}}$ and $\gamma$ for the curved interface $\left(\gamma_{\mathrm{D}}\right.$ for nanodroplet interface and $\gamma_{\mathrm{B}}$ for nanobubble interface) can be regarded as the effect of curvature on $\gamma(\Delta \gamma)$, which is

$$
\Delta \gamma_{\mathrm{D}}=\gamma_{\mathrm{D}}-\gamma_{\mathrm{F}}
$$

for nanodroplet cases and

$$
\Delta \gamma_{\mathrm{B}}=\gamma_{\mathrm{B}}-\gamma_{\mathrm{F}}
$$

for nanobubble cases.

The results for the effect of curvature on $\gamma$ are shown in Fig. 11 (a) and (b). As we can see, when the effect of $\rho_{\mathrm{G}} / \rho_{\mathrm{L}}$ on $\gamma$ is corrected, the effect of the curvature on $\gamma$ for both compressed nanodroplets and nanobubbles follows the uncompressed trends. Moreover, it is shown that the curvature shows an opposite effect on $\gamma_{\mathrm{D}}$ and $\gamma_{\mathrm{B}}$. The same method applied here was used to obtain the effect of curvature on $\delta(\Delta \delta)$, which is

$$
\Delta \delta_{\mathrm{D}}=\delta_{\mathrm{D}}-\delta_{\mathrm{F}}
$$


for nanodroplet cases and

$$
\Delta \delta_{\mathrm{B}}=\delta_{\mathrm{B}}-\delta_{\mathrm{F}}
$$

for nanobubble cases.

The results for the effect of curvature on $\delta$ are shown in Fig. 11 (c) and (d). It is shown that, as with the results for $\gamma$, by subtracting the flat interfaces value of $\delta$ from both compressed nanodroplet and nanobubble cases, it can be made to agree with the uncompressed trends. Similarly, the curvature presents opposite effects on $\delta_{\mathrm{D}}$ and $\delta_{\mathrm{B}}$. The absolute values of Tolman length may change using a different definition of pressure, which might be a factor in the Tolman length not tending to zero in Fig $11 \mathrm{c})$ and d). This will constitute the subject of further future investigations.

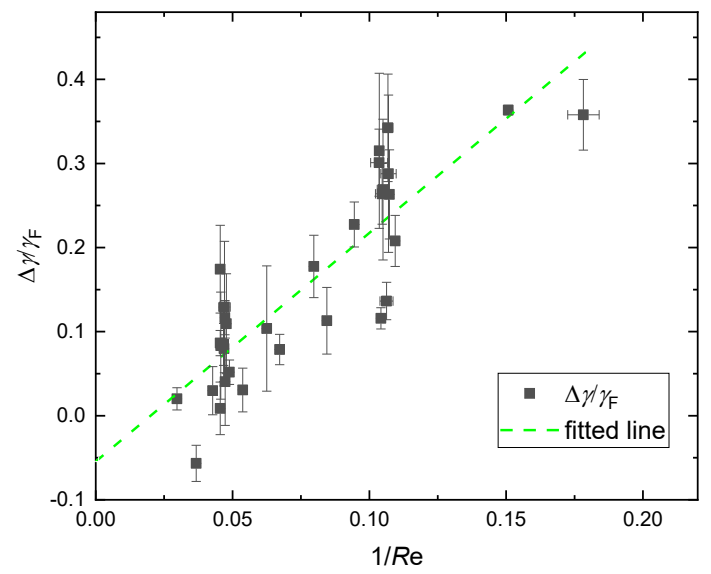

(a)

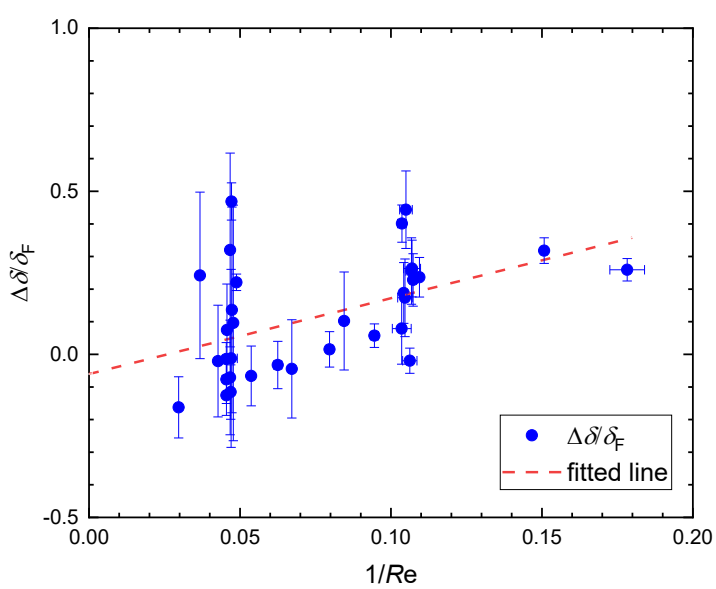

(b)

FIG. 12. Effect of curvature on (a) the surface tension and (b) Tolman length.

Figure 12 (a) shows $\Delta \gamma$ normalised by $\gamma_{\mathrm{F}}$ with the same $\rho_{\mathrm{G}} / \rho_{\mathrm{L}}$ (note here that opposite values to the results for nanodroplet interfaces are used). We can see that $\Delta \gamma / \gamma_{\mathrm{F}}$ increases with the curvature of the interfaces. As the effect of curvature is expected to fade away when the curvature decreases, a linear relationship is fitted to the curvature dependence of the surface tension. The data points agree well with the fitted line as the effect of $\rho_{\mathrm{G}} / \rho_{\mathrm{L}}$ is corrected. The results scatter evenly around the fitted line within the error bars. The effect of curvature on the surface tension vanishes at a curvature of around 0.02 where the fitted line intersects the $x$ axis. Figure 12 (b) shows $\Delta \delta$ normalised by $\delta_{\mathrm{F}}$ with the same $\rho_{\mathrm{G}} / \rho_{\mathrm{L}}$ (note here that opposite values of the results for nanobubble interfaces are used). Similar to the surface tension, a linear relationship is fitted to the Tolman length. The data points do not agree as well as that for the surface tension with the fitted line. The reason is that the relative uncertainty of the Tolman length for the flat interface case is high ${ }^{11,24}$ (as shown in Fig. 7 (b)) and the linear fit to Tolman length and $\rho_{\mathrm{G}} / \rho_{\mathrm{L}}$ for flat interface has a big possible variation.

As the effects of $\rho_{\mathrm{G}} / \rho_{\mathrm{L}}$ and curvature on $\Delta \gamma$ are investigated separately, the results for surface tension in Fig. 10 can be discussed more thoroughly here. For the uncompressed nanobubble cases, on the one hand, the pressure in the bulk liquid region is the saturated liquid-vapour pressure as there is a vapour 
region above the bulk-liquid region. However, inside the nanobubbles the pressure follows the YoungLaplace law and it increases when $R_{e}$ decreases which causes the density inside the nanobubble increase, while the density of the liquid stays relatively the same. Then $\rho_{\mathrm{G}} / \rho_{\mathrm{L}}$ increases and leads to a decrease of the surface tension as $R_{e}$ decreases. On the other hand, the curvature also has an effect on the surface tension which leads to an increase of the surface tension as $R_{e}$ decreases. When $R_{e}$ is larger than 20 , the effect of $\rho_{\mathrm{G}} / \rho_{\mathrm{L}}$ dominates, and the surface tension shows a slight decrease as $R_{e}$ decreases. When $R_{e}$ is smaller than 20 , the effect of curvature dominates, and the surface tension shows an increasing trend as $R_{e}$ decreases. For the uncompressed nanodroplet cases, there are no gas molecules in the vapour region and the pressure there is the saturated liquid vapour pressure. While inside the nanodroplets, the pressure follows the Young-Laplace law and increases as $R_{e}$ decreases. However, because the liquid is incompressible, its density stays relatively constant. Thus $\rho_{\mathrm{G}} / \rho_{\mathrm{L}}$ does not change for the nanodroplet cases and there is only the effect of the curvature. This is why the surface tension of the nanodroplet cases decreases monotonously as $R_{e}$ decreases. Similar effects can also be seen from the results for Tolman length in Fig. 10.

\section{E. Fitted Tolman equation considering both effects of density ratio and curvature}

The relationship between $\gamma$ and $\delta$ for cylindrical nanodroplet (Eq. (15)) and nanobubble (Eq. (16)) interfaces were obtained following the methods adopted by Tolman.

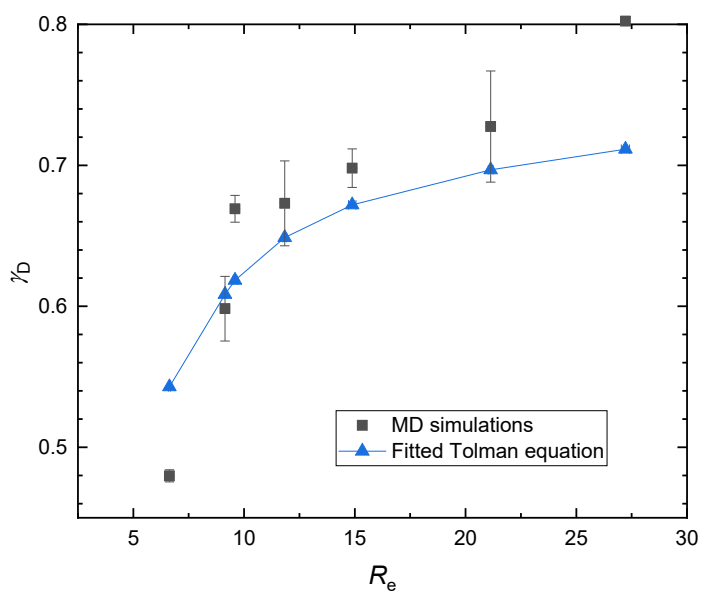

(a)

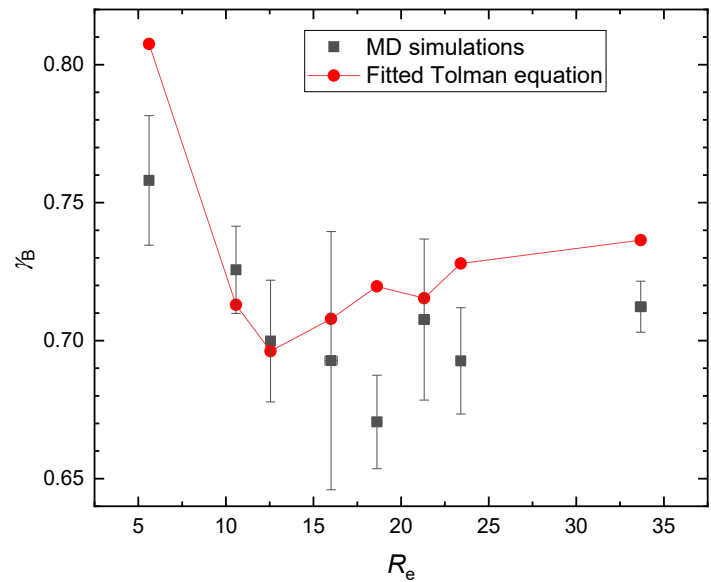

(b)

FIG. 13. Surface tension for (a) nanodroplets and (b) nanobubbles obtained from MD simulations without correction and the (fitted) Tolman equation for interfaces with different curvature.

By substituting the fitted surface tension (Eq. (22)) and Tolman length (Eq. (23)) for the flat interface, considering the effect of $\rho_{\mathrm{G}} / \rho_{\mathrm{L}}$, into Eqs. (15) and (16), we obtain the fitted Tolman equations for cylindrical nanodroplets and nanobubbles, respectively:

$$
\gamma_{\mathrm{D} R_{s}}=\frac{R_{S}\left(-1.3352 \frac{\rho_{\mathrm{G}}}{\rho_{\mathrm{L}}}+0.7695\right)}{R_{S}+\left(3.6941 \frac{\rho_{\mathrm{G}}}{\rho_{\mathrm{L}}}+1.6605\right)}
$$




$$
\gamma_{\mathrm{B} R_{S}}=\frac{R_{s}\left(-1.3352 \frac{\rho_{\mathrm{G}}}{\rho_{\mathrm{L}}}+0.7695\right)}{R_{S}-\left(3.6941 \frac{\rho_{\mathrm{G}}}{\rho_{\mathrm{L}}}+1.6605\right)}
$$

With the fitted Tolman equations, $\gamma$ for nanodroplet and nanobubble cases with a certain curvature radius and $\rho_{\mathrm{G}} / \rho_{\mathrm{L}}$ were obtained. The results are compared with the MD simulations in Fig. 13. It is shown that $\gamma$ obtained with the MD simulations and the fitted Tolman equation show similar trends for both nanodroplet and nanobubble interfaces. When the curvature radius is larger than 10 for nanodroplets and 12 for nanobubbles, $\gamma$ obtained with the two methods agree relatively well. For smaller curvature radius, $\gamma$ obtained with the fitted Tolman equation tends to be higher than that obtained with MD simulations. Such difference at the small curvature radius might come from the assumption that $\delta$ is constant, while from the results shown earlier in this study, we know that it varies with curvature radius when the curvature radius is smaller than 20. Equations (15) and (16) are obtained by integrating Eqs. (13) and (14) with respect to $R$ from infinite (flat interface) to the radius of interest $\left(R_{S}\right)$. Thus, the effect of the curvature dependence of Tolman length will accumulate when the curvature radius is smaller than 20 . This causes the higher values in the fitted Tolman equation for curvature radius smaller than around 10 for nanodroplets and around 12 for nanobubbles. 


\section{CONCLUSIONS}

In this study, we have presented a detailed comparison of nanobubbles and nanodroplets. Positive values for the Tolman length are obtained for flat, nanobubble and nanodroplet interfaces with MD simulations using the Kirkwood-Buff method. The Tolman length is defined as the distance from the surface of tension to the equimolar surface, assuming the convention that the direction from liquid to gas is positive. The effect of the gas/liquid density ratio is parameterised by measuring the surface tension and Tolman length for the case of a flat interface. It is found that the surface tension decreases linearly while the Tolman length increases linearly with the gas/liquid density ratio. Fittings are obtained which parameterise both surface tension and Tolman length as a function of density ratio. Next, the surface tension and Tolman length for nanobubbles and nanodroplets confined to solid surfaces with alternate hydrophobic and hydrophilic patterns are simulated for various curvature radii. Using the density ratio relations from the flat interface case, the effect of gas/liquid density ratio is removed and the curvature dependence of the surface tension and Tolman length for cylindrical nanobubble and nanodroplet interfaces were isolated and compared. The curvature started to show an effect when the radius of the equimolar surface is smaller than 20. The droplet and bubble show equal but opposite trends in both surface tension and Tolman length as a function of radius. Using the effect of gas/liquid density ratio obtained from flat interface cases, a fitted Tolman equation can be obtained to predict the surface tension of a curved interface with certain curvature radius and gas/liquid density ratio. Results showed that the surface tension obtained with the fitted Tolman equation are comparable with those obtained with the MD simulations. They agree well, except the higher values obtained using the fitted Tolman equation at smaller curvature radius, attributed to the accumulation of the curvature dependence of the Tolman length. Our results on the similarity and difference between droplets and bubbles, the density and curvature dependence of the surface tension and the Tolman length have important implications for nanoscale processes linked to bubble nucleation, droplet formation, wetting, moving contact line and their relevant technological applications.

\section{ACKNOWLEDGMENTS}

The project was funded by the CSC Imperial Scholarship (Grant no. 201700260048). The authors also acknowledge the support received by the Engineering and Physical Sciences Research Council (EPSRC) via Professor Dini's Established Career Fellowship, EP/N025954/1, the National Natural Science Foundation of China (grant no. 51879218, 52071272) and Natural Science Basic Research Program of Shaanxi (Program No.2020JC-18). All data are available on request by emailing the corresponding author or tribology@imperial.ac.uk. 


\section{REFERENCES}

1. T. Ito, H. Lhuissier, S. Wildeman, andD. Lohse, "Vapor bubble nucleation by rubbing surfaces: Molecular dynamics simulations," Phys. Fluids 26, 032003 (2014).

2. S. Prestipino, A. Laio, andE. Tosatti, "Shape and area fluctuation effects on nucleation theory," The Journal of Chemical Physics 140, 094501 (2014).

3. M. Foroutan, F. Esmaeilian, andT. Rad Morteza, "The change in the wetting regime of a nanodroplet on a substrate with varying wettability: A molecular dynamics investigation," Phys. Fluids 33, 032017 (2021).

4. X. Wu, N. Phan-Thien, X.-J. Fan, andT. Y. Ng, "A molecular dynamics study of drop spreading on a solid surface," Phys. Fluids 15, 1357 (2003).

5. E. R. Smith, P. E. Theodorakis, R. V. Craster, andO. K. Matar, "Moving Contact Lines: Linking Molecular Dynamics and Continuum-Scale Modeling," Langmuir 34, 12501 (2018).

6. W. Ren, andW. E, "Boundary conditions for the moving contact line problem," Phys. Fluids 19, 022101 (2007).

7. J. S. Rowlinson, "Statistical thermodynamics of small systems and interlaces," Pure and Applied Chemistry 59, 15 (1987).

8. Y. A. Lei, T. Bykov, S. Yoo, andX. C. Zeng, "The Tolman length: Is it positive or negative?," Journal of the American Chemical Society 127, 15346 (2005).

9. H. M. Lu, andQ. Jiang, "Size-dependent surface tension and Tolman's length of droplets," Langmuir 21, 779 (2005).

10. A. E. van Giessen, andE. M. Blokhuis, "Direct determination of the Tolman length from the bulk pressures of liquid drops via molecular dynamics simulations," The Journal of chemical physics 131, 164705 (2009).

11. $\varnothing$. Wilhelmsen, D. Bedeaux, andD. Reguera, "Tolman length and rigidity constants of the Lennard-Jones fluid," The Journal of chemical physics 142, 064706 (2015).

12. T. Young, "An essay on the cohesion of fluids," Abstracts of the Papers Printed in the Philosophical Transactions of the Royal Society of London 1, 171 (1832).

13. R. C. Tolman, "The effect of droplet size on surface tension," The journal of chemical physics 17, 333 (1949).

14. R. C. Tolman, "The superficial density of matter at a liquid - vapor boundary," The journal of chemical physics 17, 118 (1949).

15. T. V. Bykov, andX. C. Zeng, "A patching model for surface tension and the Tolman length," The Journal of chemical physics 111, 3705 (1999).

16. A. A. Onischuk, P. A. Purtov, A. M. Baklanov, V. V. Karasev, andS. V. Vosel, "Evaluation of surface tension and Tolman length as a function of droplet radius from experimental nucleation rate and supersaturation ratio: Metal vapor homogeneous nucleation," The Journal of chemical physics 124, 014506 (2006).

17. Y.-Q. Xue, X.-C. Yang, Z.-X. Cui, andW.-P. Lai, "The effect of microdroplet size on the surface tension and Tolman length," The Journal of Physical Chemistry B 115, 109 (2011).

18. N. Bruot, andF. Caupin, "Curvature dependence of the liquid-vapor surface tension beyond the Tolman approximation," Physical review letters 116, 056102 (2016).

19. P. Montero de Hijes, J. R. Espinosa, V. Bianco, E. Sanz, andC. Vega, "Interfacial Free Energy and Tolman Length of Curved Liquid-Solid Interfaces from Equilibrium Studies," The Journal of Physical Chemistry C 124, 8795 (2020).

20. S. S. Rekhviashvili, E. V. Kishtikova, R. Y. Karmokova, andA. M. Karmokov, "Calculating the Tolman constant," Technical Physics Letters 33, 48 (2007).

21. P. Rehner, andJ. Gross, "Surface tension of droplets and Tolman lengths of real substances and mixtures from density functional theory," The Journal of chemical physics 148, 164703 (2018).

22. P. Rehner, A. Aasen, andl. Wilhelmsen, "Tolman lengths and rigidity constants from free-energy functionals -- General expressions and comparison of theories," 151, 244710 (2019). 
23. S. Burian, M. Isaiev, K. Termentzidis, V. Sysoev, andL. Bulavin, "Size dependence of the surface tension of a free surface of an isotropic fluid," Phys. Rev. E 95, 062801 (2017).

24. F. Calvo, "Molecular dynamics determination of the surface tension of silver-gold liquid alloys and the Tolman length of nanoalloys," The Journal of Chemical Physics 136, 154701 (2012).

25. A. Malijevský, andG. Jackson, "A perspective on the interfacial properties of nanoscopic liquid drops," Journal of Physics: Condensed Matter 24, 464121 (2012).

26. A. Ghoufi, P. Malfreyt, andD. J. Tildesley, "Computer modelling of the surface tension of the gasliquid and liquid-liquid interface," Chemical Society Reviews 45, 1387 (2016).

27. R. Lovett, andM. Baus, "A molecular theory of the Laplace relation and of the local forces in a curved interface," The Journal of chemical physics 106, 635 (1997).

28. J. W. Gibbs, The collected Works of J. Willard Gibbs, Vol. 1 (Longmans, Green and Co., New York, 1928).

29. F. O. Koenig, "On the thermodynamic relation between surface tension and curvature," The Journal of Chemical Physics 18, 449 (1950).

30. S. Plimpton, "FAST PARALLEL ALGORITHMS FOR SHORT-RANGE MOLECULAR-DYNAMICS," Journal of Computational Physics 117, 1 (1995).

31. K. P. Travis, B. D. Todd, andD. J. Evans, "Departure from Navier-Stokes hydrodynamics in confined liquids," Physical Review E Statistical Physics Plasmas Fluids \& Related Interdisciplinary Topics 55, 4288 (1997).

32. S. Maheshwari, M. van der Hoef, X. Zhang, andD. Lohse, "Stability of Surface Nanobubbles: A Molecular Dynamics Study," Langmuir 32, 11116 (2016).

33. F. Varnik, J. Baschnagel, andK. Binder, "Molecular dynamics results on the pressure tensor of polymer films," The Journal of Chemical Physics 113, 4444 (2000).

34. D. M. Heyes, E. R. Smith, D. Dini, andT. A. Zaki, "The equivalence between volume averaging and method of planes definitions of the pressure tensor at a plane," The Journal of chemical physics 135, 024512 (2011).

35. B. D. Todd, D. J. Evans, andP. J. Daivis, "Pressure tensor for inhomogeneous fluids," Phys. Rev. E 52, 1627 (1995).

36. E. R. Smith, andC. Braga, "Hydrodynamics Across a Fluctuating Interface," arXiv preprint arXiv:2007.10779 (2020).

37. M. P. Moody, andP. Attard, "Curvature-dependent surface tension of a growing droplet," Physical review letters 91, 056104 (2003). 


\section{SUPPORTING INFORMATION}

\section{A. Deduction of the Tolman equations for cylindrical interfaces.}

For a cylindrical interface, the equations for surface tension based on pressure tensors can be deduced following the method similar to that for a spherical interface. The system can be simplified as a cylindrical drop of phase $\alpha$ immersed in phase $\beta$ within a cylindrical coordinate system $r, \theta, z$. The pressure tensor of the system is:

$$
\boldsymbol{P}=P_{\mathrm{N}}\left(\boldsymbol{e}_{r} \boldsymbol{e}_{r}\right)+P_{\mathrm{T}}\left(\boldsymbol{e}_{\theta} \boldsymbol{e}_{\theta}\right)+P_{z}\left(\boldsymbol{e}_{z} \boldsymbol{e}_{z}\right)
$$

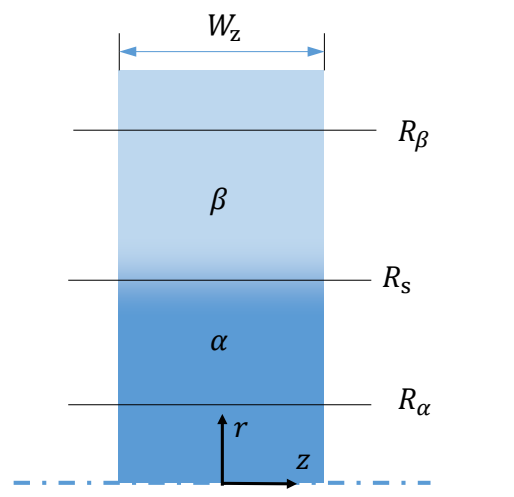

FIG. A.1. Stress state of a strip in a longitudinal plane of a cylindrical interface.

Then, consider a longitudinal strip with a width of $W_{z}$ in the $z$ direction, stretching from $R_{\alpha}$ (in the bulk $\alpha$ region) to $R_{\beta}$ (in the bulk $\beta$ region) in the $r$ direction, at a constant $\theta$ (shown in Fig. A.1). The surface of tension is at $R_{S}$ in $r$ direction. The force acting on one side of the strip can be expressed in two ways: as an integral of $P_{\mathrm{T}}(r)$ over the strip, or, as a result of the pressure $P_{\alpha}$ acting on the surface between $R_{\alpha}$ and $R_{S}$, of the pressure $P_{\beta}$ acting on the surface between $R_{S}$ and $R_{\beta}$, and of the surface tension $\gamma$ acting at $R_{S}$. The two expressions of the force and the momentum of the force about $z$ axis equal each other:

$$
\int_{R_{\alpha}}^{R_{\beta}} r^{n} W_{z} P_{\mathrm{T}} d r=\int_{R_{\alpha}}^{R_{S}} r^{n} W_{z} P_{\alpha} d r+\int_{R_{S}}^{R_{\beta}} r^{n} W_{z} P_{\beta} d r+R_{S}{ }^{n} W_{z} \gamma
$$

where $n=0$ and 1 represent the force acting on the strip and its momentum about $z$ axis, respectively. Here we define a pressure $P_{\alpha, \beta}\left(r ; R_{S}\right)$ which equals $P_{\alpha}$ for $r$ between $R_{\alpha}$ and $R_{S}$ and $P_{\beta}$ for $r$ between $R_{S}$ and $R_{\beta}$. Then Eq. (A.2) can be rewrote as:

$$
\gamma=\int_{R_{\alpha}}^{R_{\beta}}\left(\frac{r}{R_{S}}\right)^{n}\left(P_{\alpha, \beta}\left(r ; R_{S}\right)-P_{\mathrm{T}}\right) d r
$$

At the same time, from the hydrostatic equilibrium condition of the system $\boldsymbol{\nabla} \cdot \boldsymbol{P}=0$ we have:

$$
\int_{R_{\alpha}}^{R_{\beta}} \frac{d r^{m} P_{\mathrm{N}}}{d r} d r=\int_{R_{\alpha}}^{R_{\beta}} r^{m-1}\left[(m-1) P_{\mathrm{N}}+P_{\mathrm{T}}\right] d r
$$

where $m$ is an integer. Integrate the left side of Eq. (A.4): 


$$
R_{\beta}{ }^{m} P_{\beta}-R_{\alpha}{ }^{m} P_{\alpha}=\int_{R_{\alpha}}^{R_{\beta}} r^{m-1}\left[(m-1) P_{\mathrm{N}}+P_{\mathrm{T}}\right] d r
$$

In the meanwhile, form the integration of $m r^{m-1} P_{\alpha, \beta}\left(r ; R_{S}\right)$ :

$$
\begin{gathered}
\int_{R_{\alpha}}^{R_{\beta}} m r^{m-1} P_{\alpha, \beta}\left(r ; R_{s}\right) d r=\int_{R_{\alpha}}^{R_{s}} m r^{m-1} P_{\alpha} d r+\int_{R_{s}}^{R_{\beta}} m r^{m-1} P_{\beta} d r \\
=R_{\beta}{ }^{m} P_{\beta}-R_{\alpha}{ }^{m} P_{\alpha}+\left(P_{\alpha}-P_{\beta}\right) R_{S}{ }^{m}
\end{gathered}
$$

Substitute Eq. (A.5) into (A.6):

$$
\left(P_{\alpha}-P_{\beta}\right) R_{S}{ }^{m}=\int_{R_{\alpha}}^{R_{\beta}}\left(m r^{m-1} P_{\alpha, \beta}\left(r ; R_{S}\right)-r^{m-1}\left[(m-1) P_{\mathrm{N}}+P_{\mathrm{T}}\right]\right) d r
$$

At the surface of tension we have:

$$
P_{\alpha}-P_{\beta}=\frac{\gamma}{R_{S}}
$$

Substitute Eq. (A.8) into (A.7):

$$
\gamma=\int_{R_{\alpha}}^{R_{\beta}}\left(m\left(\frac{r}{R_{S}}\right)^{m-1} P_{\alpha, \beta}\left(r ; R_{S}\right)-\left(\frac{r}{R_{S}}\right)^{m-1}\left[(m-1) P_{\mathrm{N}}+P_{\mathrm{T}}\right]\right) d r
$$

Equation (A.3) times 2 for $\mathrm{n}=1$ minus Eq. (A.9) for $\mathrm{m}=2$ :

$$
\gamma=\int_{R_{\alpha}}^{R_{\beta}} \frac{r}{R_{S}}\left(P_{\mathrm{N}}-P_{\mathrm{T}}\right) d r
$$

Equation (A.10) divided by Eq. (A.9) for $\mathrm{m}=0$ :

$$
R_{S}{ }^{2}=\frac{\int_{R_{\alpha}}^{R_{\beta}} r\left(P_{\mathrm{N}}-P_{\mathrm{T}}\right) d r}{\int_{R_{\alpha}}^{R_{\beta}} \frac{1}{r}\left(P_{\mathrm{N}}-P_{\mathrm{T}}\right) d r}
$$


B. Examples of the $\gamma$ and $\delta$ for nanodroplet interfaces calculated with two procedures.

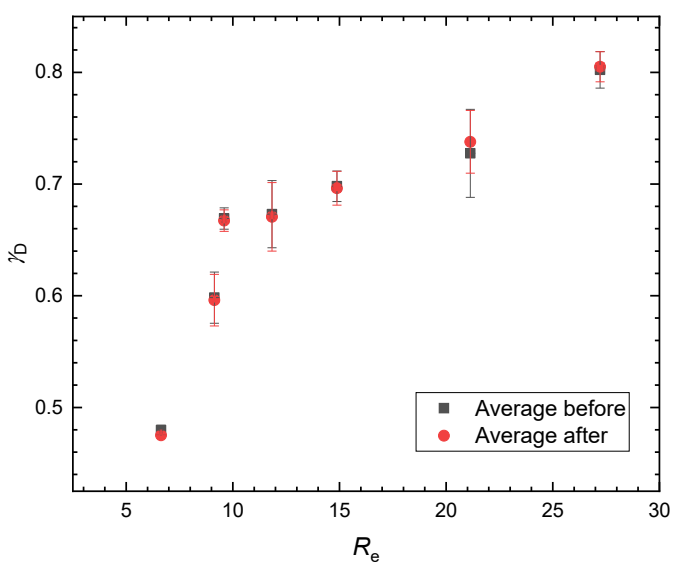

(a)

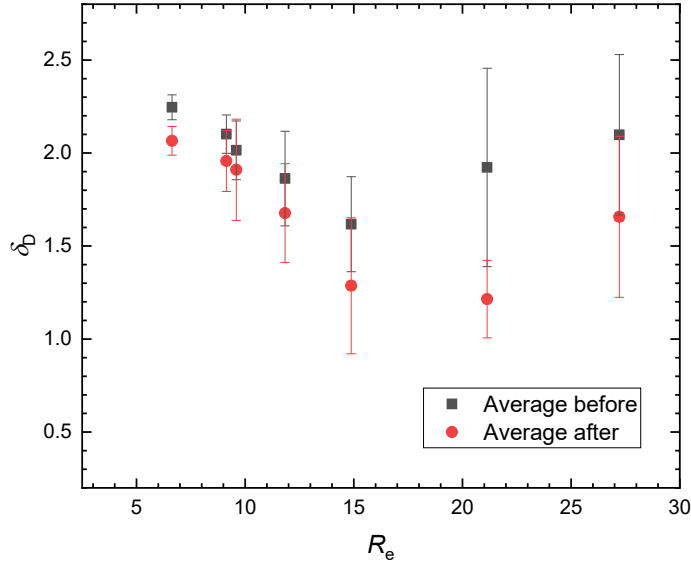

(b)

FIG. B.1. The surface tension for nanodroplet cases depicted as $\gamma_{\mathrm{D}}$ (a) and the Tolman length for nanodroplet cases depicted as $\delta_{\mathrm{D}}$ (b) calculated with different procedures. 